\title{
Template Cross-Linking Effects on Morphologies of Swellable Block Copolymer and Mesostructured Silica Thin Films
}

\author{
Ryan C. Hayward, ${ }^{\dagger}$ Bradley F. Chmelka, ${ }^{\dagger}$ and Edward J. Kramer, ${ }^{* \dagger, \dagger}$ \\ Department of Chemical Engineering and Materials Department, University of California, \\ Santa Barbara, California 93106
}

Received October 27, 2004; Revised Manuscript Received February 26, 2005

\begin{abstract}
Mesostructured silica films were formed from cross-linked poly $\left(d_{8}\right.$-styrene-block-2-vinylpyridine) (dPS- $b$-P2VP) diblock copolymer template films by swelling with silica precursor solutions. The mesoscopic and macroscopic morphologies of the resulting materials depended strongly on the extent of P2VP cross-linking. Without cross-linking, extensive rearrangement of the initial 2D hexagonal arrangement of dPS cylinders took place during swelling, leading to wormlike mesostructures. At low extents of template cross-linking, osmotic stresses led to a surface-wrinkling instability with a morphology directed by the anisotropic mechanical properties of the swelled block copolymer mesostructure. At higher degrees of P2VP cross-linking, wrinkling was suppressed, and the composite silica/polymer mesostructures corresponded to essentially affine expansions of the initial template structures normal to the film plane. At low extents of P2VP cross-linking, the mesostructures of the hybrid block copolymer/silica films were preserved during oxidation and removal of the block copolymer template species, leading to stable mesoporous silica films. At high extents of P2VP cross-linking, however, insufficient amounts of silica were incorporated to form stable porous inorganic materials, leading to mesostructural collapse. An intermediate level of cross-linking allowed the preparation of stable mesoporous silica films with structures that closely resembled those of the initial template films.
\end{abstract}

\section{Introduction}

A recently developed strategy for the preparation of templated mesoporous inorganic films involves the introduction of sol-gel precursors into preformed block copolymer films. ${ }^{1-3}$ Selective incorporation and condensation of the precursors in one component of the block copolymer generates an ordered inorganic/organic hybrid mesostructure that is subsequently converted to a mesoporous film by oxidation and removal of the polymer template. This technique offers the potential for greater flexibility in processing conditions than previous approaches to the formation of mesoporous inorganic oxides, where organization of the template species occurs simultaneously with formation of the inorganic component. ${ }^{4-6}$ By separating the self-assembly of the template molecules from the incorporation of the inorganic component, the mesostructure of the block copolymer film can first be manipulated by, e.g., the application of electric fields, ${ }^{7,8}$ topographical patterning of the substrate, ${ }^{9}$ or solvent annealing 10 to achieve the desired phase, mesostructural alignment, and/or long-range ordering. The preorganized film can subsequently be converted into a porous inorganic material with a mesostructure that replicates that of the initial template. This approach is anticipated to yield improved control over the morphology of templated mesostructured inorganic films.

In a previous report, we showed that cross-linking of the preformed block copolymer film is important to the preservation of mesostructural ordering during swelling and incorporation of inorganic sol-gel precursors. ${ }^{3}$ Without cross-links, the block copolymer template rearranges extensively upon swelling, ${ }^{2,3}$ destroying the

\footnotetext{
$\dagger$ Department of Chemical Engineering.

$\$$ Materials Department.

* Corresponding author. E-mail: edkramer@mrl.ucsb.edu.
}

spatial patterning imposed by preprocessing. By introducing cross-links, however, it is possible to restrict rearrangement of the block copolymer during swelling and thereby produce inorganic oxide films with mesostructures that closely mimic those of the template films. Naturally, the extent to which the template film is cross-linked is an important variable in determining the morphology of the resulting composite material, since it determines how extensively the film can be swelled.

When a polymeric material is placed in contact with solvent, entropy of mixing provides an osmotic driving force for solvent molecules to swell the polymer. If the polymer is cross-linked, swelling requires that the crosslinked polymer strands be stretched away from their initial Gaussian distribution of chain segments, providing an entropic resistance to expansion of the network. The ultimate extent of swelling is achieved when forces produced by the osmotic pressure are balanced by those arising from chain stretching. A widely applied approach to describing the swelling of polymeric gels is the Flory-Rehner theory. ${ }^{11}$ For an isotropic, neutral polymer gel at low extents of cross-linking, the equilibrium volumetric degree of swelling of the network is predicted to scale as the density of cross-links to the $-3 / 5$ power. For isotropic materials, this swelling occurs equally in all dimensions, with the linear extent of swelling in any one Cartesian direction predicted to scale as the density of cross-links to the $-1 / 5$ power.

In the case of thin polymer gels attached to rigid substrates, the polymer network is unable to expand in the directions parallel to the plane of the substrate. Flory-Rehner theory predicts that in this case the linear extent of swelling in the direction normal to the film plane should be larger than the linear swelling of an equivalent unconfined gel, scaling as the density of cross-links to the $-1 / 3$ power. This result has been 
qualitatively verified by experiments. ${ }^{12}$ The expansion occurs only in one dimension, however, so that the overall volumetric swelling of a thin gel confined to a surface is many times less than in the unconfined case. ${ }^{12-14}$ Thus, there remains a strong osmotic driving force for swelling of the confined polymer network in the dimensions parallel to the plane of the film, putting the film into a state of biaxial compressive stress. Such swelling stresses can be quite large and have been observed to give rise to mechanical instabilities that lead to wrinkling of the free surface of confined gels ${ }^{15}$ and delamination of thin polymer layers from their supporting substrates. ${ }^{16-18}$ However, as the degree of crosslinking increases and the extent of swelling decreases, the magnitude of these swelling stresses is also diminished.

The behavior of the cross-linked block copolymer template films considered here is more complex due to their anisotropic mesostructures and correspondingly anisotropic mechanical properties. The charged nature of the poly(2-vinylpyridine) component under the acidic conditions employed and the relatively large extents of film cross-linking add further complications to the simple Flory-Rehner treatment outlined above. However, the swelling behavior of these materials can be qualitatively understood in the same basic manner as homogeneous polymer gels. If the polymer template film is heavily cross-linked, the structure will only dilate slightly when placed in contact with solvent. In this case, the swelled network can incorporate only a relatively small volume of inorganic precursors, which may not allow formation of a robust mesoporous material. By contrast, if the film is lightly cross-linked, extensive swelling of the structure can accommodate larger volumes of inorganic precursor species. However, if the degree of cross-linking is too low, substantial deformation of the mesostructure may occur due to the influence of osmotic swelling stresses. It is necessary to optimize the extent of template cross-linking to obtain wellordered and robust mesostructured inorganic solids.

Here, we investigate the effect of extent of crosslinking on the morphologies of hybrid silica/block copolymer films formed from poly $\left(d_{8}\right.$-styrene-block-2vinylpyridine) (dPS- $b$-P2VP) template films with initial thicknesses of $\sim 100-200 \mathrm{~nm}$. The films were first thermally annealed, giving rise to well-ordered structures consisting of cylinders of dPS oriented parallel to the film plane. Chemical cross-linking of the majority P2VP component was then carried out, followed by swelling of the films with an aqueous solution of silica precursors to produce mesostructured silica/block copolymer composite materials. For lightly cross-linked films, osmotic stresses induced a surface-wrinkling instability similar to that observed by Tanaka et al. ${ }^{15}$ during swelling of confined acrylamide gels. The morphology of the wrinkling observed here was found to be dictated by the defect texture of the block copolymer and the length scale by the thickness and extent of crosslinking of the film. For more extensively cross-linked template films, the block copolymer structure swelled affinely to incorporate the sol-gel precursor species. If cross-linking was too extensive, only small amounts of inorganic material were incorporated, and the structure appeared to collapse upon removal of the block copolymer template. In the regime of intermediate crosslinking, it was possible to produce hybrid silica/polymer films with mesostructures that were related to those of the starting block copolymer template via a nearly affine expansion normal to the film plane and incorporated sufficient amounts of the condensable inorganic components such that removal of the block copolymer template yielded a stable mesoporous silica film.

In addition to the surface-wrinkling instability, the osmotic stresses generated during swelling of a thin polymer film can also lead to delamination of the film from its supporting substrate, if the adhesion at this interface is overcome. ${ }^{17,18}$ This gives rise to a mechanical instability wherein the polymer film buckles away from the substrate. We use the term "buckling" to refer to this instability, which is distinct from the case of "wrinkling" discussed above, in which texturing of the surface occurs with the film remaining anchored to the substrate. In the current work, we found that swelling of cross-linked dPS- $b$-P2VP films could lead to delamination and buckling of sufficiently thick films. The morphology of buckles that formed just above the minimum thickness required for delamination was dictated by the anisotropic mechanical properties of the block copolymer film, as similarly observed for the surface wrinkles. The onset of buckling could be shifted to greater film thicknesses by functionalization of the substrate surface to promote adhesion of the polymer film. This suggests that, while film buckling is an important mode of failure to consider, it is a problem that can be effectively overcome.

\section{Experimental Section}

Sample Preparation. The poly $\left(d_{8}\right.$-styrene-block-2-vinylpyridine) block copolymer template was synthesized by standard anionic polymerization techniques. ${ }^{19}$ sec-Butyllithium was used to initiate the polymerization of $d_{8}$-styrene in THF at $-78{ }^{\circ} \mathrm{C}$, followed by the sequential addition of 2-vinylpyridine. Monomers and solvent were purchased from Aldrich and purified as described previously. ${ }^{19}$ Termination was accomplished via the addition of degassed butanol, and the polymer was precipitated in hexane, collected via filtration, and dried. A small amount of dPS homopolymer, which formed due to chain termination upon addition of the $2 \mathrm{VP}$ monomer, was removed by reprecipitation of the polymer from THF into cyclohexane. Size-exclusion chromatography (SEC) indicated that the homopolymer was removed by reprecipitation and that the diblock copolymer had an approximate molecular weight of $\sim 30 \mathrm{~kg} / \mathrm{mol}$. However, adsorption of the P2VP component to the SEC column made for unreliable determination of molecular weights, which instead were established by matrixassisted laser desorption/ionization time-of-flight mass spectrometry (MALDI-MS). The polymer was dissolved at $0.2 \mathrm{mg} /$ $\mathrm{mL}$ in chloroform and mixed with a solution of $34 \mathrm{mg} / \mathrm{mL}$ dithranol in chloroform and a saturated solution of sodium chloride in ethanol in a volumetric ratio of 2:1:2. ${ }^{20}$ MALDIMS indicated a molecular weight of $33.3 \mathrm{~kg} / \mathrm{mol}$ with a polydispersity index $\left(M_{\mathrm{w}} / M_{\mathrm{n}}\right)$ of 1.01 . The molar composition was determined to be $25 \%$ dPS from solution-state ${ }^{13} \mathrm{C}$ NMR. This corresponds to a number-average degree of polymerization of dPS $79-b-\mathrm{P} 2 \mathrm{VP}_{239}$. P2VP homopolymer $\left(M_{\mathrm{n}}=35 \mathrm{~kg} / \mathrm{mol}\right)$ was purchased from Aldrich and used as received.

To suppress osmotic-stress-induced film buckling, the silicon substrates were functionalized with surface amine groups to improve adhesion of the polymer films. Silicon substrates were first cleaned by UV/ozone treatment and placed in a desiccator with $2 \mathrm{~g}$ of 3-(aminopropyl)triethoxysilane, evacuated to 50 Torr, and left for at least $15 \mathrm{~h}$ to provide a surface coating of aminosilane. The wafers were then sonicated for $10 \mathrm{~min}$ in acetone and rinsed with ethanol to remove excess silane. Thin films of dPS- $b$-P2VP were prepared by spin-coating from a toluene solution containing $2.5-4.0 \mathrm{wt} \%$ polymer, depending on the desired thickness. Annealing of bulk and thin film samples was carried out under high vacuum $\left(<10^{-6}\right.$ Torr $)$ at 
a temperature between 210 and $240{ }^{\circ} \mathrm{C}$ for at least $12 \mathrm{~h}$. The lattice spacing of the block copolymer decreased slightly with increased annealing temperature, but no order-order or order-disorder transitions were observed over this temperature range. Thin films of P2VP homopolymer were spin-coated from 2.5 to $4.0 \mathrm{wt} \%$ solutions in pyridine. Typically, the homopolymer samples were not annealed, though similar phenomena were observed for unannealed samples and samples annealed in the same manner as the block copolymer films. Cross-linking of the P2VP component of block copolymer and homopolymer films was accomplished by placing films into a desiccator evacuated to 50 Torr with $100 \mu \mathrm{L}$ of 1,4-diiodobutane. The desiccator was then placed in a forced-convection oven maintained at $60 \pm 2{ }^{\circ} \mathrm{C}$.

Silica was incorporated into the dPS- $b$-P2VP or P2VP homopolymer films by immersing the films in an acidic $(\mathrm{pH}$ 0) solution containing $25 \mathrm{mM}$ of prehydrolyzed silica precursor species for $12-15 \mathrm{~h}$ at $20^{\circ} \mathrm{C}$. The films were subsequently rinsed with deionized water. The silica precursor solution was formed by mixing $2.7 \mathrm{~mL}$ of $1.0 \mathrm{M} \mathrm{HCl}$ (aqueous) with $10 \mu \mathrm{L}$ of tetramethoxysilane for $\sim 1 \mathrm{~min}$ before immersing the polymer films. To generate mesoporous silica films, the block copolymer/silica hybrid films were treated in a UV/ozone cleaner (UVOCS) for $1 \mathrm{~h}$ to oxidize and thereby remove the polymer template species. Alternatively, the block copolymer species were removed by calcination in air by heating the films at $1{ }^{\circ} \mathrm{C} / \mathrm{min}$ to $400{ }^{\circ} \mathrm{C}$ and holding for $4 \mathrm{~h}$.

Characterization. Small-angle X-ray scattering was performed at the Stanford Synchrotron Radiation Laboratory on Beamline 4-2, with X-rays of wavelength $\lambda=1.38 \AA$ and a sample-detector distance of $2512 \mathrm{~mm}$ (calibrated with silver behenate). A 2D CCD detector was used, but the scattering was isotropic, so the data are presented as an azimuthally averaged powder pattern, with intensity plotted in terms of the scattering vector $q=4 \pi \sin (\theta) / \lambda$, where $2 \theta$ is the total scattering angle. Transmission electron microscopy (TEM) was performed on annealed bulk copolymer samples cut into $\sim 200$ $\mathrm{nm}$ thick slices using room temperature ultramicrotomy. Sliced film sections were kept dry, as floating onto water produced breakage, due to swelling of P2VP. Ultramicrotomed sections were retrieved on a copper TEM grid and stained by exposure to iodine vapor for $\sim 3 \mathrm{~h}$ prior to imaging. Iodine selectively stained the P2VP component of the polymer, providing contrast for TEM. Cross-sectional samples of thin films for TEM were prepared by focused ion-beam milling using an FEI DB235 system. Prior to cross-sectioning, a layer of carbon $(\sim 50 \mathrm{~nm}$ thick) was deposited on the film surface to help reduce beam damage to the sample. Plan-view TEM samples of block copolymer/silica thin films were prepared by allowing a drop of $35 \mathrm{wt} \%$ aqueous poly(acrylic acid) (PAA) to dry on the film surface. Removal of the PAA caused the film to detach from the substrate, and the film could subsequently be freed by floating at an air-water interface as the PAA dissolved. ${ }^{21}$ TEM imaging was performed using a FEI Tecnai G2 Sphera microscope, operated at $200 \mathrm{kV}$.

Deuterium and carbon depth profiling of annealed thin films were accomplished by secondary ion mass spectrometry (SIMS) using a Physical Electronics 6650 dynamic SIMS system. A beam of $\mathrm{O}_{2}{ }^{+}$ions accelerated through $2 \mathrm{kV}$ was rastered across the film surface to form a $300 \mu \mathrm{m}$ crater, with negative secondary ions collected. Films were prepared on a silicon wafer coated with $250 \mathrm{~nm}$ of silicon oxide to minimize drift of the signals due to charging effects. To determine the in-plane morphology of the block copolymer film, a subsequent SIMS etch was halted at the first maximum in the deuterium signal, corresponding to the mid-plane of the first layer of dPS cylinders. A slight difference in the speed with which dPS and P2VP were etched by the oxygen gun of the SIMS gave rise to a height contrast between the blocks that allowed imaging via scanning force microscopy (SFM). ${ }^{9}$ SFM height images were collected in SIMS craters as well as on the unetched surfaces of polymer/silica hybrid films, using a Digital Instruments multimode Nanoscope IIIa SFM instrument operated in tapping mode. Surface texturing of polymer/silica hybrid films was also analyzed by field-emission scanning electron microscopy
(SEM) on samples coated with gold-palladium, using an FEI XL40 Sirion microscope operating with an accelerating voltage of $3 \mathrm{kV}$.

The degrees of quaternization of the cross-linked block copolymer and P2VP homopolymer films were measured via X-ray photoelectron spectroscopy (XPS) using a Kratos Axis Ultra XPS system with a monochromated aluminum X-ray source. Nitrogen 1s binding energy spectra were chargereferenced such that the carbon 1s peak was centered at 285.0 $\mathrm{eV}$. The relative concentrations of quaternized and nonquaternized pyridine units were determined by fitting Gaussian curves to the peaks for the two components. The uncertainties in the reported values are $\pm 0.3-0.5 \%$ for dPS- $b$-P2VP samples and $\pm 0.2 \%$ for P2VP homopolymer samples. To determine the degree of functionalization at the bottom surface, films were spin-cast onto sodium chloride crystals and annealed and cross-linked as usual. Dissolution of the salt crystal in water (at neutral $\mathrm{pH}$ ) freed the cross-linked film to float at the airwater interface. The polymer film was subsequently retrieved onto a silicon wafer with the surface that was originally adjacent to the substrate now at the free surface.

Thicknesses of dry and swelled block copolymer thin films, silica/block copolymer hybrid films, and mesoporous silica films were measured via phase-modulated ${ }^{22}$ variable-angle ellipsometry, using a Beaglehole picometer ellipsometer. Measurements were performed with a $\mathrm{He}-\mathrm{Ne}$ laser (wavelength, $\lambda=$ $632.8 \mathrm{~nm}$ ), with incident and reflected angles that varied from $40^{\circ}$ to $70^{\circ}$ (measured from the normal to the substrate) for films supported on silicon wafers (refractive index, $n=3.88-$ $0.018 i$ ). For measurements of films in contact with water, samples were placed into a cylindrical glass cell filled with 17 $\mathrm{mL}$ of water $(n=1.33)$ containing $\mathrm{HCl}$ at one of the following concentrations: $10^{-4}, 10^{-3}, 10^{-2}, 10^{-1}$, or $10^{0} \mathrm{M}^{23}$

The degree of swelling was measured for a given film in order of increasing acid concentration, and the cell was thoroughly washed with deionized (Milli-Q) water before measuring the swelling of the next film. Measurements were typically performed 1-2 min after placing the film in contact with aqueous solution, and each measurement required about $4 \mathrm{~min}$. In several cases measurements were repeated a second time directly after the first measurement, and no significant variation in the ellipsometric profiles were observed, indicating that the degree of swelling of the film was not changing on a time scale of 5-10 min.

The ellipsometer measures the real and imaginary parts of the reflectivity ratio $\rho$, where

$$
\rho=\frac{r_{\mathrm{p}}}{r_{\mathrm{s}}}=\tan (\Psi) \mathrm{e}^{i \Delta}
$$

where $r_{\mathrm{p}}$ and $r_{\mathrm{s}}$ represent the complex reflection coefficients of $\mathrm{p}$ - and s-polarized light, respectively, and $\psi$ and $\Delta$ are the angles that are typically used for representing ellipsometric data. ${ }^{24,25}$ Fitting was carried out using the measured values for the imaginary component of $\rho$, since these were found to give more consistent results in the presence of the solution cell than the real component. Values of film thickness and refractive index were determined by fitting the ellipsometric data to a simple model of a single layer of nonabsorbing, isotropic material as described by Azzam and Bashara, ${ }^{24}$ using a Levenberg-Marquardt nonlinear least-squares fitting routine, with reported parameter uncertainties corresponding to the $95 \%$ confidence intervals of the fits. This procedure was found to yield reasonable fits to the data, and thus no additional parameters were introduced into the model.

To verify oxidation of the block copolymer templates by UV/ ozone treatment and calcination, films were prepared on silicon wafers polished on both sides, and Fourier transform infrared (FTIR) absorption spectra were recorded in transmission geometry. Information on the elemental composition of silica/ block copolymer and mesoporous silica films was provided by XPS. To remove surface contamination and provide information on the composition of the film interior, samples were 
etched to a depth of $\sim 20 \mathrm{~nm}$ prior to analysis using an argon ion gun in the XPS chamber.

\section{Results and Discussion}

Structure of dPS-b-P2VP in Bulk and Thin Films. The bulk morphology of the block copolymer was investigated by small-angle X-ray scattering (SAXS) and transmission electron microscopy (TEM), as shown in Figure 1. The SAXS data show a primary scattering peak $\left(q^{*}\right)$ at $0.306 \pm 0.001 \mathrm{~nm}^{-1}$, as well as a pronounced peak at $\sqrt{ } 3 q^{*}$ and a weaker peak at $\sqrt{ } 7 q^{*}$. The presence of scattering peaks at these spacings indicates that the polymer forms a $2 \mathrm{D}$ hexagonal lattice of dPS cylinders with a lattice parameter $a=23.7 \pm 0.1 \mathrm{~nm}$. No peak is observed at the expected position of $\sqrt{ } 4 q^{*}$, presumably due to the small value of the form factor at this value of $q$ for the cylinders that give rise to the observed scattering. The dotted line in Figure 1a represents the calculated form factor for constant-density cylinders with radius $r:{ }^{26}$

$$
P(q) \propto\left(\frac{J_{1}(q r)}{q r}\right)^{2}
$$

where $J_{1}$ is the first-order Bessel function, using a value of $r=6.3 \mathrm{~nm}$ (the expected size of the dPS cylinders, based on the measured lattice parameter and an approximate volume fraction of $25 \% \mathrm{dPS})$. Indeed, the first zero of the form factor is at $q=0.61 \mathrm{~nm}^{-1}$, very near to the value of $\sqrt{ } 4 q^{*}\left(0.612 \mathrm{~nm}^{-1}\right)$. TEM imaging of bulk samples corroborated the assignment of the $2 \mathrm{D}$ hexagonal structure. Parts b and c of Figure 1 show micrographs taken with the electron beam nearly perpendicular and nearly parallel to the long axes of the dPS cylinders, respectively. Measurement of the $d_{10}$ spacing from the TEM micrographs yields a lattice parameter of $a=24 \pm 1 \mathrm{~nm}$, which is in good agreement with the value determined by SAXS.

The morphology of the dPS-b-P2VP block copolymer in thin films was investigated by using a combination of dynamic secondary-ion mass spectrometry (SIMS) and scanning force microscopy (SFM). As shown in Figure 2a, SIMS depth profiling of an annealed film shows an oscillation of the deuterium $\left({ }^{2} \mathrm{H}^{-}\right)$signal, corresponding to organization of the block copolymer mesostructure with the (10) planes of the $2 \mathrm{D}$ hexagonal structure parallel to the film plane. The carbon $\left({ }^{12} \mathrm{C}^{-}\right)$ signal is essentially constant throughout the thickness of the film but drops off rapidly when the film has been etched through to the underlying silicon substrate. The deuterium signal is a maximum at the free surface, indicating that dPS forms a wetting layer at the top (free) surface of the film. The deuterium SIMS signal in Figure 2a shows another four peaks below the surface, indicating that this sample consisted of four layers of dPS cylinders. At the silicon substrate, the deuterium signal is close to its minimum value, due to the wetting of this interface by the P2VP block.

Comparison of SIMS depth profiles with film thicknesses measured via ellipsometry revealed a spacing between layers of cylinders of $20 \pm 0.5 \mathrm{~nm}$, in agreement with the bulk value of $d_{10}(20.5 \mathrm{~nm})$. To satisfy this spacing and the wetting conditions at the interfaces, the block copolymer adopts film thicknesses $(l)$ of

$$
l=l_{\mathrm{w}}+n d_{10}
$$

where $n$ is the number of layers of dPS cylinders and $l_{\mathrm{w}}$
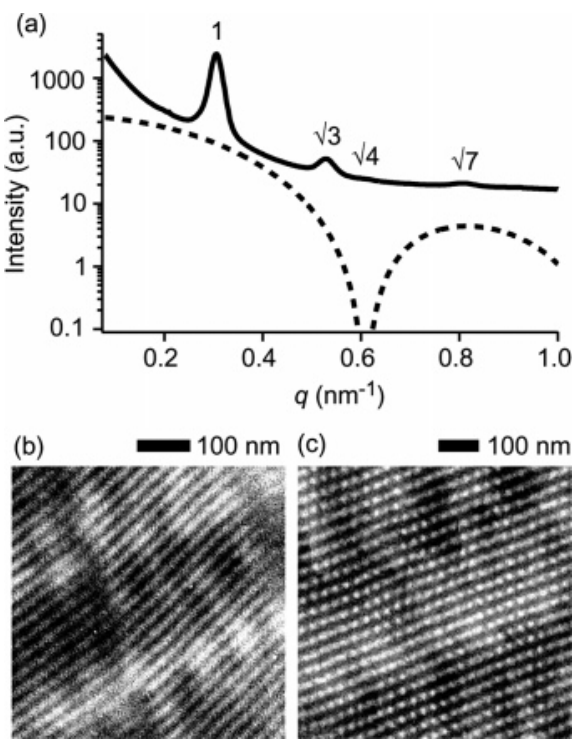

Figure 1. (a) SAXS data from a bulk sample of dPS- $b$-P2VP block copolymer indicating a $2 \mathrm{D}$ hexagonal structure. The dotted line represents the form factor calculated for a cylinder with a radius of $6.3 \mathrm{~nm}$. (b) TEM image of the bulk 2D hexagonal structure with the electron beam nearly perpendicular to the long axes of the dPS cylinders. (c) TEM image with the electron beam nearly parallel to the long axes of the dPS cylinders.

is the thickness of the wetting layer at the free interface $(10 \pm 1 \mathrm{~nm})$. Preparation of films with initial thicknesses not equal to these discrete values led to lateral separation into regions of different thicknesses, forming islands or holes. The in-plane morphology of the block copolymer films was determined by halting a SIMS etch at the first subsurface maximum of the deuterium signal, corresponding to the mid-plane of the first layer of dPS cylinders. SFM imaging of the etched surface (Figure 2b) showed the characteristic "fingerprint" defect pattern of cylinder-forming block copolymers with microdomains oriented parallel to the film plane.

Cross-Linking of dPS-b-P2VP Thin Films. Previous work has shown that cross-linking of the block copolymer template film is crucial to the preservation of mesostructural ordering upon swelling and infiltration of inorganic precursors. ${ }^{3}$ Since the minority dPS block in the polymer template used here is glassy $\left(T_{\mathrm{g}}\right.$ of bulk polystyrene $\sim 100{ }^{\circ} \mathrm{C}$ ), the individual cylindrical microdomains of the polymer remain intact during swelling, even in the absence of cross-links. Thus, to limit structural reorganization of the polymer template, it is sufficient to cross-link the majority P2VP phase. For other systems where deformation of the minority component also occurs during swelling, it will likely be important to cross-link both components of the block copolymer template species.

Chemical cross-linking was carried out by treatment with 1,4-diiodobutane (DIB) vapor, which quaternizes the pyridine rings of the P2VP component. ${ }^{27}$ To determine the degree of quaternization, X-ray photoelectron spectroscopy (XPS) was conducted on the thin film samples. Figure $3 \mathrm{a}$ shows the nitrogen $1 \mathrm{~s}$ region of the XPS spectrum for a non-cross-linked film and for a sample cross-linked for $10.5 \mathrm{~h}$. In the case of the noncross-linked sample, a single peak is observed at 399.1 $\mathrm{eV}$ (Figure 3a, bottom), corresponding to the binding energy of pyridine $\mathrm{N}$ 1s electrons. ${ }^{28}$ Following crosslinking via DIB vapor treatment, a second peak corre- 

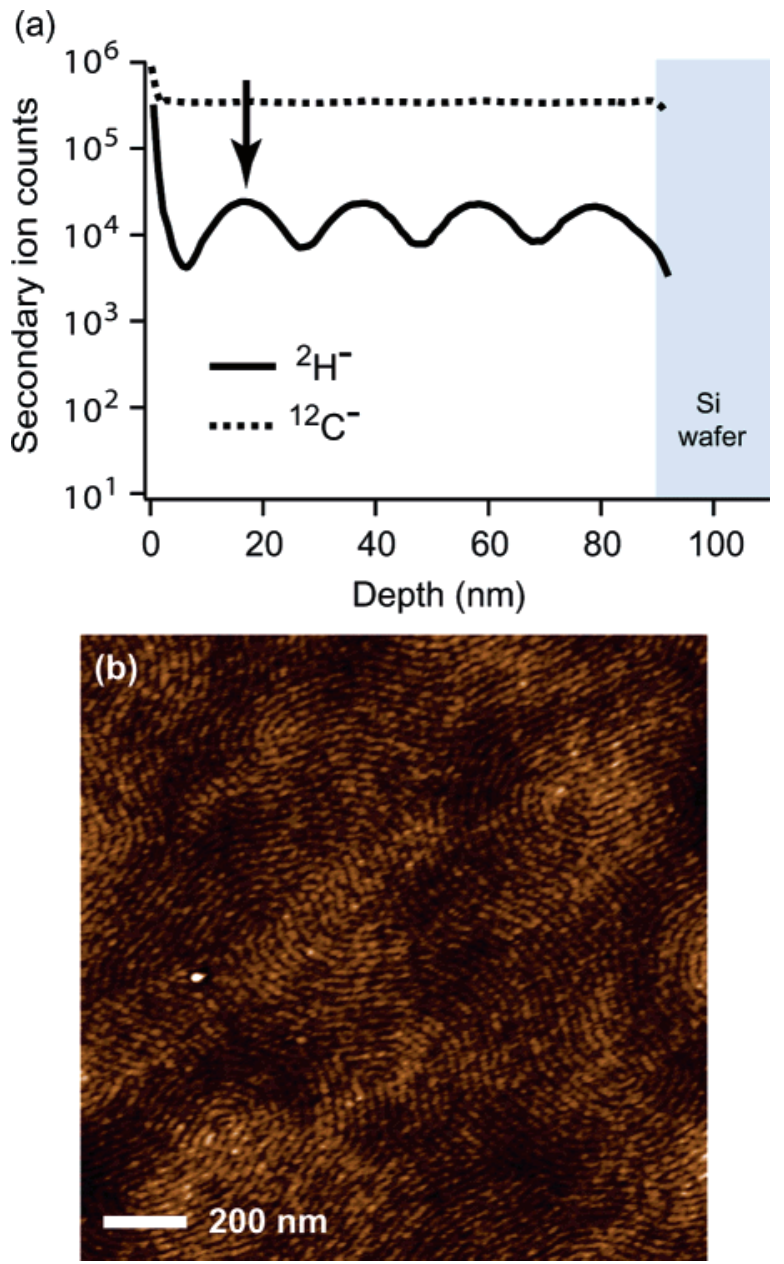

Figure 2. (a) SIMS depth profile of a dPS- $b$-P2VP thin film (thickness $90 \mathrm{~nm}$ ) showing a thin wetting layer of dPS at the top surface and 4 layers of dPS cylinders below. (b) An SFM height image of the dPS- $b$-P2VP block copolymer film showing the characteristic morphology of cylinders oriented parallel to the film plane. Prior to imaging, the film was etched via SIMS to the midplane of the first layer of dPS cylinders, as indicated by the arrow in the SIMS depth profile. Light areas are higher than dark, with a total height scale of $5 \mathrm{~nm}$.

sponding to quaternized pyridine is seen at $402.2 \mathrm{eV}$ (Figure 3a, top). The relative concentrations of quaternized and nonquaternized pyridine groups were determined by fitting Gaussian curves to the observed peaks (shown as dotted lines in Figure 3a, top). The degree of quaternization increased with time of exposure to DIB in a nearly linear fashion, as shown in Figure $3 \mathrm{~b} .^{29}$

Since XPS is exclusively sensitive to species within several nanometers of the top surface of a sample, it was important to ensure that the measured functionalization accurately reflected the degree of cross-linking throughout the film thickness. Previously, SIMS depth profiling was used to show that the iodine content was nearly constant throughout the thickness of a crosslinked dPS- $b$-P2VP film, indicating uniform incorporation of the cross-linking agent DIB. ${ }^{3}$ In addition to the SIMS experiments, XPS was performed on both the free and substrate interfaces of cross-linked block copolymer films by preparing samples on sodium chloride substrates and subsequently freeing the films by floating them on an air-water interface. Typically, the bottom surface showed a lower degree of quaternization than the top by $2-5 \%$, but this difference did not increase with film thickness. Thus, the difference more likely
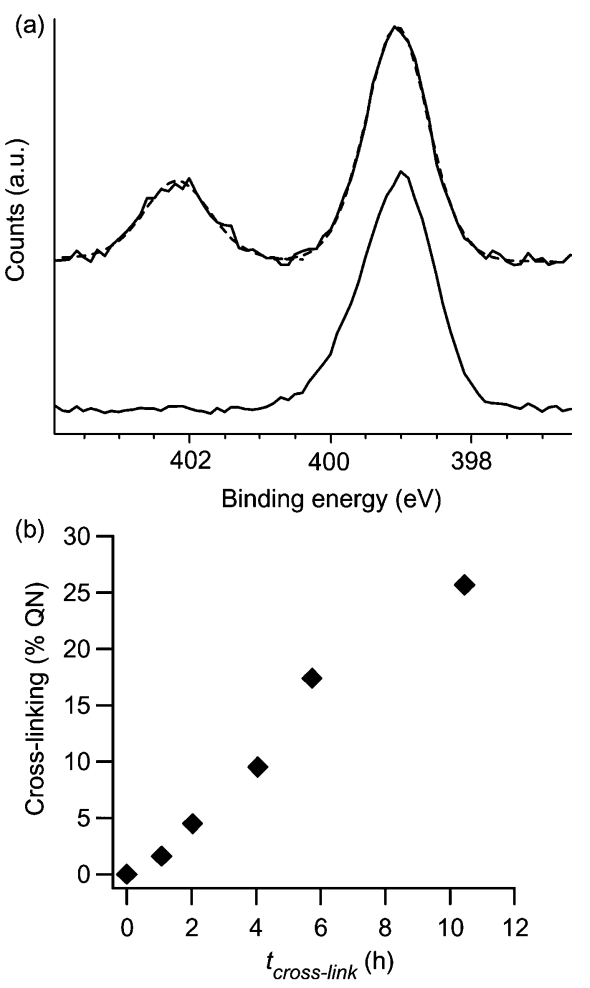

Figure 3. (a) Nitrogen 1s regions of XPS spectra for dPS- $b$ P2VP films (bottom) before cross-linking and (top) following $10.5 \mathrm{~h}$ of exposure to DIB vapor. The dotted lines represent Gaussian fits to the two components of the spectrum for the cross-linked film. (b) The extent of cross-linking (expressed as percent P2VP quaternization, \% QN) as determined from XPS as a function of time of exposure to DIB vapor. The uncertainty in the measured values is $\pm 0.3-0.5 \%$, which is less than the size of the data points.

reflects a lower reactivity of pyridine units interacting with the substrate rather than limitations on the mass transport of the DIB cross-linking agent through the film.

Cross-linking of the dPS- $b$-P2VP films resulted in increased film thicknesses due to incorporation of the cross-linking agent DIB. Table 1 summarizes the thicknesses of a series of films cross-linked to different extents, as measured by variable angle ellipsometry. Based on SIMS depth profiles and measurements of film thicknesses, a slight expansion of the block copolymer structure normal to film plane occurred upon incorporation of DIB. SIMS depth profiles and SFM images nearly identical to those in Figure 2 were observed for crosslinked films,${ }^{3}$ indicating that the cross-linking procedure does not otherwise alter the morphology of the block copolymer.

Swelling of Cross-Linked Films. To investigate how the cross-linked block copolymer films swell when in contact with aqueous solutions, $90 \mathrm{~nm}$ thick films (corresponding to four layers of dPS cylinders) were prepared with different extents of P2VP cross-linking. The thicknesses of these films in air or submerged in water containing different concentrations of $\mathrm{HCl}$ were measured by variable-angle spectroscopic ellipsometry. Profiles were fitted according to a model of a single nonabsorbing dielectric layer, ${ }^{24}$ with the film thickness and refractive index as adjustable parameters. Figure 4 shows the measured ratio of swelled film thickness to dry film thickness $\left(l_{\text {swelled }} / l_{\text {dry }}\right)$ as a function of $\mathrm{pH}$ of the swelling solution for block copolymer films with four different extents of cross-linking. At $\mathrm{pH} 4$ or greater, 
Table 1. Film Thicknesses $(l)$ and Refractive Indices $(n)$ Determined from Variable-Angle Ellipsometric Measurements on dPS-b-P2VP Block Copolymer, Block Copolymer/Silica, and Mesoporous Silica Films for Different Extents of P2VP Quaternization (\% QN)

\begin{tabular}{|c|c|c|c|c|c|c|c|}
\hline \multirow[b]{2}{*}{$\% \mathrm{QN}^{a}$} & \multicolumn{2}{|c|}{ dPS- $b-\mathrm{P} 2 \mathrm{VP}$} & \multicolumn{2}{|c|}{ dPS- $b$-P2VP/silica } & \multicolumn{3}{|c|}{ mesoporous silica } \\
\hline & $l(\mathrm{~nm})$ & $n$ & $l(\mathrm{~nm})$ & $n$ & $l(\mathrm{~nm})$ & $n$ & $\operatorname{porosity}^{b}(\%)$ \\
\hline 0 & $89.7 \pm 0.9$ & $1.587 \pm 0.003$ & & & & & \\
\hline 4.0 & $94.9 \pm 0.1$ & $1.603 \pm 0.001$ & $167.6 \pm 0.2$ & $1.509 \pm 0.0015$ & $93.9 \pm 0.1$ & $1.361 \pm 0.001$ & 18.6 \\
\hline 10.5 & $100.3 \pm 0.1$ & $1.644 \pm 0.001$ & $152.6 \pm 0.9$ & $1.577 \pm 0.009$ & $72.9 \pm 0.1$ & $1.387 \pm 0.001$ & 13.4 \\
\hline 19.5 & $112.3 \pm 0.1$ & $1.697 \pm 0.001$ & $139.6 \pm 0.5$ & $1.653 \pm 0.006$ & $45.6 \pm 0.1$ & $1.427 \pm 0.002$ & 5.5 \\
\hline
\end{tabular}

${ }^{a}$ Extents of quaternization measured by XPS with uncertainties of $\pm 0.5 \%$. ${ }^{b}$ Porosity is calculated from the refractive indices, assuming that the pores are filled with air $(n=1)$ and taking a value of $n=1.456$ for the silica matrix. This represents an approximate lower bound for the porosity due to the adsorption of atmospheric water by the samples. ${ }^{47}$

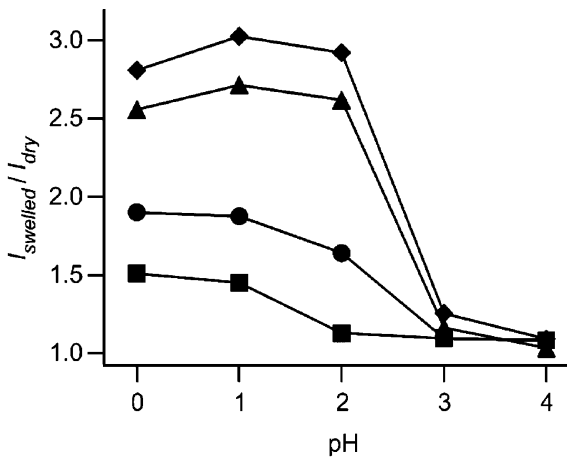

Figure 4. Ratios of swelled film thickness to dry film thickness $\left(l_{\text {swelled }} / l_{\text {dry }}\right)$ measured by using variable-angle ellipsometry as functions of $\mathrm{pH}$ for dPS- $b$-P2VP films with different extents of quaternization: $5.3 \%(\bullet), 7.0 \%(\boldsymbol{\Delta}), 17.5 \%(\bullet), 27.4 \%$ (ם). Uncertainties in the ratios derived from fitting the ellipsometric data are smaller than the size of the data points.

the films were swelled only slightly, $\sim 10 \%$ over their dry thicknesses, as water is a poor solvent for neutral P2VP. At or below $\mathrm{pH}$ 2, however, the pyridine groups of P2VP become protonated, ${ }^{30}$ and the polymer films swelled more extensively, consistent with water being a good solvent for the polyelectrolyte P2VP-H $\mathrm{H}^{+}$. Under such conditions, the film thicknesses were considerably increased over the dry state, and the amount of swelling could be adjusted by changes in the extent of crosslinking. At $\mathrm{pH} 1$, the swelled thickness varied from 3.0 times the dry state at 5.3\% P2VP quaternization to only 1.45 times the dry thickness at $27.4 \%$ quaternization.

From the ellipsometric data, it is apparent that, in order to swell films sufficiently for the incorporation of inorganic species, the $\mathrm{pH}$ of the swelling solution should be 2 or below. Furthermore, some insight can gained about the optimal degree of cross-linking, since the amount that the polymer film expands is expected to place an upper limit on the amount of inorganic material that can be incorporated. The volume fraction of polymer within the $\mathrm{P} 2 \mathrm{VP} /$ water domain $\left(\phi_{\mathrm{P} 2 \mathrm{VP}}\right)$ can be calculated as

$$
\phi_{\mathrm{P} 2 \mathrm{VP}}=\frac{l_{\mathrm{dry}}-l_{\mathrm{dPS}}}{l_{\mathrm{swelled}}-l_{\mathrm{dPS}}}
$$

where $l_{\text {swelled }}$ is the measured thickness of the swelled cross-linked film, $l_{\mathrm{dry}}$ is the measured thickness prior to swelling, and $l_{\mathrm{dPS}}$ is the portion of the dry thickness made up by the dPS component (estimated to be 0.25 times the thickness of a dry non-cross-linked film). For example, for the film cross-linked $5.3 \%$ at $\mathrm{pH} 0$, the swelling ratio $l_{\text {swelled }} / l_{\text {dry }}=2.81$ corresponds to a value of $\phi_{\mathrm{P} 2 \mathrm{VP}}=0.27$. This large degree of swelling means that there is ample volume associated with the swelled P2VP hydrogel for the incorporation of inorganic precursor species. Conversely, at $27.4 \%$ quaternization, the film swelled to only 1.51 times its dry thickness at the same $\mathrm{pH}$, corresponding to $\phi_{\mathrm{P} 2 \mathrm{VP}}=0.60$. As will be shown below, the amount of silica that can be incorporated into such a highly cross-linked film is insufficient to allow formation of a robust inorganic network in the swelled P2VP component.

Morphologies of Hybrid Silica/Polymer Films. Incorporation of silica into the cross-linked dPS- $b$-P2VP block copolymer template films was accomplished by swelling films in an acidic ( $\mathrm{pH}$ 0) solution containing silica sol-gel precursors. Soluble silica species diffused into the hydrophilic $\mathrm{P} 2 \mathrm{VP}-\mathrm{H}^{+}$region of the polymer film, where they were sequestered and ultimately condensed to form a continuous silica network. The details of the process of silica incorporation have not yet been fully elucidated; however, this behavior is consistent with previous uses of poly(vinylpyridine) as a template for silica formation ${ }^{31-33}$ and the strong adsorption of P2VP onto silica surfaces under highly acidic conditions. ${ }^{34}$ One potential complication for the strategy used here is the capping film of dPS ( $\sim 2.5 \mathrm{~nm}$ thick $)$ at the free interface of the annealed block copolymer films. The presence of a continuous layer of glassy polymer at the free surface could impede mass transport of inorganic precursor species from solution into the continuous $\mathrm{P} 2 \mathrm{VP}$ component in the interior of the films. However, scanning electron microscopy (SEM) and SFM imaging of film surfaces after swelling in the acidic precursor solution showed that reconstruction of the top layer of the film occurred upon swelling; 10-100 $\mathrm{nm}$ wide holes were observed in the dPS layer, through which inorganic species could presumably diffuse freely. Were this not the case, steps would likely be needed to remove the surface layer of dPS or to prevent it from forming in the first place (e.g., by annealing in a solvent environment where the free surface is wetted by P2VP rather than in a vacuum).

As summarized in Table 1, thicknesses of the silica/ block copolymer hybrid films were measured by using variable-angle ellipsometry for samples prepared from template films with different extents of cross-linking. At the lowest extent of cross-linking (4.0\% quaternization), a 77\% increase in thickness occurred upon incorporation of silica into the block copolymer film. ${ }^{35} \mathrm{Ap}$ plying eq 4, and using the thickness of the hybrid film for $l_{\text {swelled, the volume fraction of P2VP in the P2VP/ }}$ silica region of this film is estimated to be $\phi_{\mathrm{P} 2 \mathrm{VP}}=0.50$, with the remainder being predominantly silica. Since half of the volume of the P2VP/silica component of this film was comprised of silica species, it was anticipated that the mesostructure of the film would survive removal of the polymer template. As discussed below, cross-sectional TEM imaging of a sample prepared from 

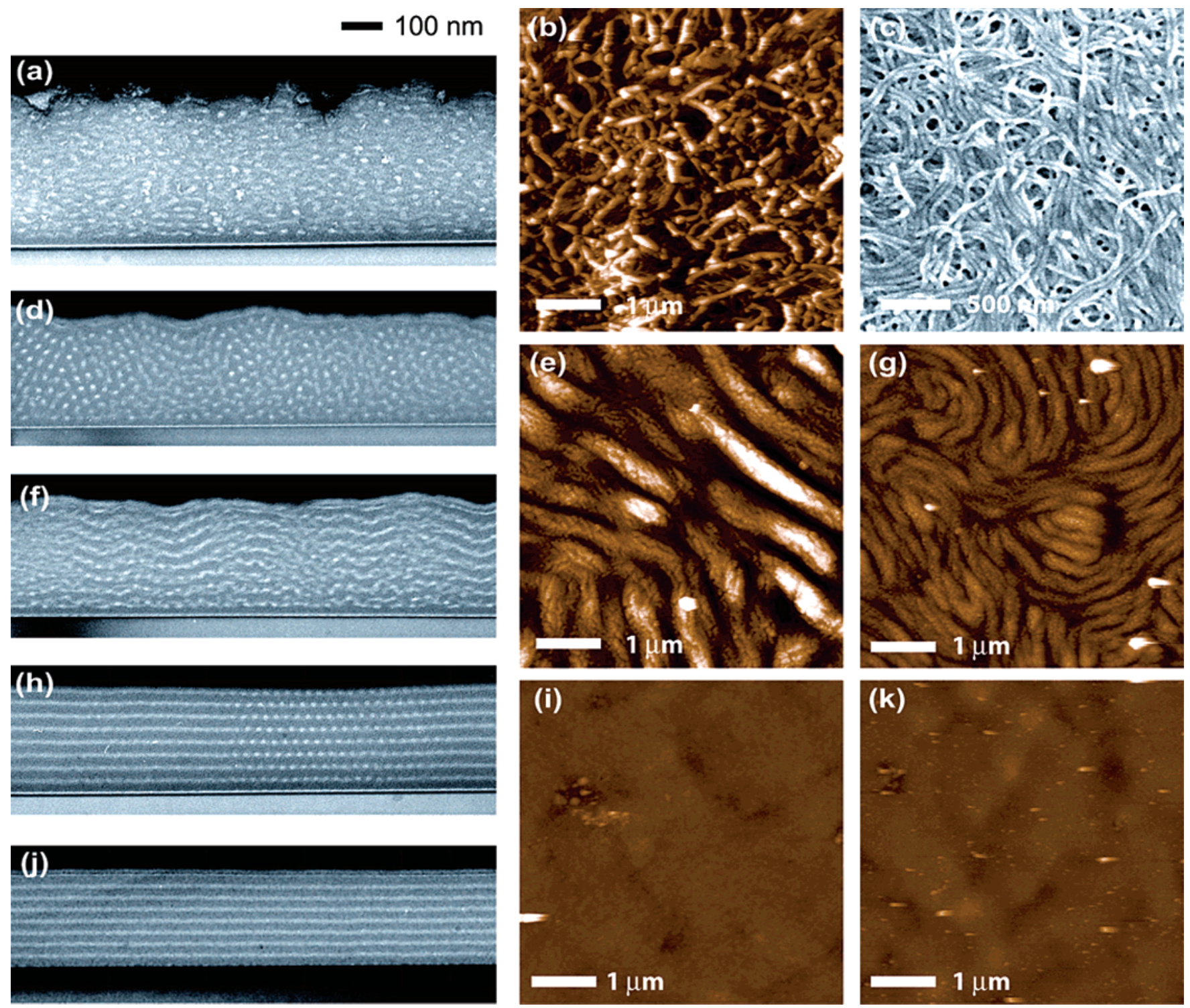

Figure 5. Cross-sectional TEM images and surface characterization (SFM, SEM) for a series of silica/dPS- $b$-P2VP films with initial template film thicknesses of $150-170 \mathrm{~nm}$ and different extents of cross-linking. (a) Cross-sectional TEM of a film formed from a non-cross-linked dPS- $b$-P2VP template and (b) corresponding SFM image of the film surface showing the reconstructed texture of the top layer of the polymer. (c) SEM image of a region of a film formed from a non-cross-linked dPS- $b$-P2VP template, where the top layer was removed during swelling, revealing the interior morphology of the film. Cross-sectional TEM and corresponding SFM images of the film surfaces for silica/dPS- $b$-P2VP films formed from templates with different degrees of quaternization: (d, e) 1.6\%, (f, g) 4.5\%, (h, i) 9.5\%, (j, k) 25.7\%. The scale bar above (a) applies to all of the cross-sectional TEM images. The SFM images show height contrast with a total scale of $150 \mathrm{~nm}$; bright areas are higher than dark areas.

a dPS- $b$-P2VP template film with a similar extent of cross-linking showed that this was indeed the case. Increasing the extent of cross-linking to $10.5 \%$ and $19.5 \%$ quaternization led to incorporation of smaller amounts of silica precursor. In these cases, the increases in film thickness upon incorporation of silica were $52 \%$ and $24 \%$, corresponding to $\phi_{\mathrm{P} 2 \mathrm{VP}}=0.60$ and 0.77 , respectively. The amount of silica incorporated thus appears to be limited by the extent to which the template film swells while in contact with the aqueous inorganic precursor solution. As demonstrated below, for the most highly cross-linked films (20-26\% quaternization), the amount of inorganic material incorporated is apparently too small to form a robust mesoporous film, with mesostructural collapse occurring upon removal of the block copolymer template.

The morphologies of the hybrid silica/polymer films also depend on the extent of cross-linking of the initial polymer template film. Previously, it was shown that, for dPS- $b$-P2VP template films consisting of four layers of dPS cylinders (initially $90 \mathrm{~nm}$ thick) with a degree of P2VP quaternization of $8-11 \%$, the block copolymer structure swelled normal to the film plane in essentially an affine manner during incorporation of silica. ${ }^{3}$ Here, mesostructural ordering in resulting hybrid dPS- $b$ $\mathrm{P} 2 \mathrm{VP} /$ silica films is correlated with extents of P2VP quaternization (cross-linking) from 0 to $26 \%$. Crosssectional TEM and SFM images of the film surfaces are shown in Figure 5 for a series of samples prepared with initial thicknesses of $150-170 \mathrm{~nm}$ (7-8 layers of cylinders) and different extents of cross-linking. Crosssectional TEM images of the sample prepared from a non-cross-linked dPS- $b$-P2VP template film (Figure 5a) show a disordered morphology, with no indication of a hexagonal arrangement of the dPS cylinders. Figure 5b is an SFM height image of the top surface of the same 
film, again showing a mesoscopically disordered morphology. In this case, the contrast is dominated by features that formed upon rearrangement of the top wetting layer during swelling. In some areas of the film, the wetting layer was completely removed during swelling, allowing the morphology of the cylinders below the film surface to be observed, as in the SEM image in Figure 5c. From this micrograph, it can be seen that in the absence of cross-linking, the individual cylinders of the block copolymer have remained intact during swelling and incorporation of silica. However, extensive rearrangement has occurred to alleviate osmotic stresses during swelling, resulting in a composite mesostructure of disordered cylinders.

By comparison, the cross-sectional TEM micrographs in Figure 5d,f of lightly cross-linked block copolymer/ silica films (1.6\% and 4.5\% P2VP quaternization, respectively) show interesting textures in regions where the long axes of the dPS cylinders are normal to the image plane. The (10) planes of cylinders, which were initially oriented parallel to the substrate in the block copolymer template film, can clearly be seen to undulate in the composite films. The SFM images of the surfaces of these films (Figure 5, e and g, respectively) show that the undulation of cylinder layers corresponds to a wrinkling of the film surfaces with characteristic lateral length scales of hundreds of nanometers. An increase in the extent of cross-linking at the same film thickness apparently leads to a decrease in both the magnitude and the characteristic length scale of this surface wrinkling.

Qualitatively, the observed distortion of cylinder layers in Figure 5d,f is similar to the mechanical instabilities that have previously been observed in mesophases undergoing uniaxial strain, namely the Helfrich-Hurault instability in smectic and columnar liquid crystals ${ }^{36}$ and the closely related instabilities in block copolymers with both lamellar and cylindrical morphologies. ${ }^{37}$ In those cases, periodic undulation of mesophase layers occurs to minimize local expansion of the characteristic spacing between layers or cylinders during strain. The morphologies result from a balance between elastic moduli that resist changes in the characteristic mesophase spacings and the bending energy of the layers or cylinders that are deformed. Above a critical threshold of strain, a mechanical instability leads to undulations that typically coarsen into a chevron pattern at larger applied strain. ${ }^{37}$

In the present case, the observed undulation of the dPS cylinder layers apparently represents a mechanical instability induced by the biaxial compressive osmotic stresses that are generated during swelling of the crosslinked thin films. ${ }^{38}$ Such stresses have previously been known to give rise to mechanical instabilities at the surfaces of laterally confined acrylamide gels ${ }^{15}$ as well as to induce delamination and buckling of polymer films when osmotic stresses overcome the adhesion of the film to the substrate. ${ }^{16-18}$ Similarly, biaxial compressive stresses in thin films that arise due to mismatches in thermal expansion are known to produce a variety of wrinkling or buckling instabilities. ${ }^{39}$ To our knowledge, the data presented here are the first example of a mechanical instability in block copolymer films induced by osmotic swelling stresses. The characteristics and nature of the instability will be considered at greater length below.

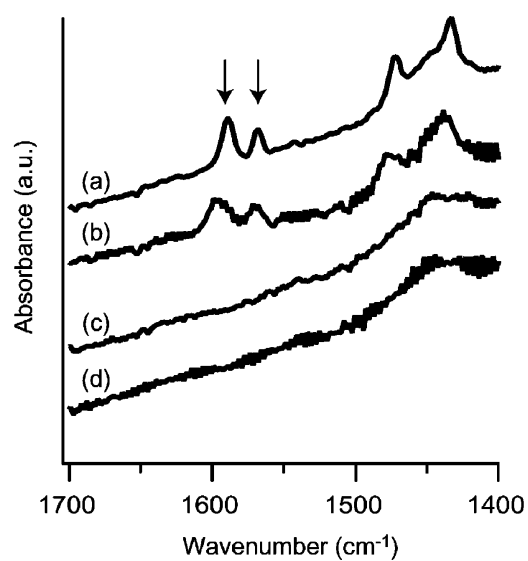

Figure 6. FTIR spectra for (a) a cross-linked dPS-b-P2VP template film, $100 \mathrm{~nm}$ thick, with 5.5\% quaternization of the P2VP moieties, (b) the composite silica/block copolymer film prepared from the template film, (c) the mesoporous silica film formed by UV/ozone treatment of the silica/block copolymer film, and (d) the mesoporous silica film formed by calcination of the silica/block copolymer film. The arrows denote the positions of the pyridine ring-stretching modes monitored to verify oxidation of the block copolymer template species.

Composite silica/block copolymer films formed from more extensively cross-linked template films are related to the initial structure of the block copolymer template via a nearly affine expansion normal to the film plane..$^{40}$ As shown in Figure 5h,j, cross-sectional TEM micrographs of $9.5 \%$ and $25.7 \%$ quaternized composite films clearly show that the (10) planes of cylinders remain parallel to the substrate. For the $9.5 \%$ quaternized film, the image in Figure 5h shows a domain with the long axes of the cylinders nearly perpendicular to the image plane. Here, it can be seen that the structure consists of a distorted 2D hexagonal arrangement of cylinders, where the spacing normal to the substrate has dilated during incorporation of silica, but the in-plane ordering has remained essentially unperturbed. For the most extensively cross-linked film (25.7\%, Figure 5j), the cross-sectional image contains no regions with the cylinders oriented normal to the image plane. However, the well-defined stripes in the image, along with separate cross-sectional SEM imaging (not shown), establish that the (10) planes of cylinders remained parallel to the substrate in this case as well. ${ }^{41}$ The SFM images in parts $i$ and $\mathrm{k}$ of Figure 5 of the surfaces of the $9.5 \%$ and $25.7 \%$ quaternized films, respectively, show that the more heavily cross-linked films are significantly flatter than the lightly cross-linked samples in Figure 5e,g. For a given film thickness, there is apparently a degree of cross-linking beyond which the wrinkling instability is suppressed. For the 150-170 nm thick template films investigated here, this point appears to be between 4.5 and $9.5 \%$ quaternization of the P2VP moieties.

Block Copolymer Template Removal. To obtain mesoporous silica films from the silica/dPS- $b$-P2VP hybrid materials, the block copolymer template species were removed via UV/ozone treatment ${ }^{42,43}$ or by calcination to $400{ }^{\circ} \mathrm{C}$. FTIR measurements, shown in Figure 6 , were used to monitor the pyridine ring-stretching modes at 1570 and $1590 \mathrm{~cm}^{-1}$ to verify oxidation of the block copolymer species. These were the most intense IR signals from the polymer template, and they permitted measurement in transmission geometry for relatively thin films $(100-200 \mathrm{~nm})$. The pyridine ringstretching modes (denoted by the arrows in Figure 6) 
Table 2. Elemental Ratios Determined from XPS Measurements of a Silica/dPS-b-P2VP Film and Mesoporous Silica Films Prepared by UV/Ozone Treatment and Calcination

\begin{tabular}{llcrr}
\hline \multicolumn{1}{c}{ sample } & \multicolumn{1}{c}{$\mathrm{C} / \mathrm{Si}$} & $\mathrm{C}$ remaining ${ }^{a}(\%)$ & $\mathrm{N} / \mathrm{Si}$ & $\mathrm{N} \mathrm{remaining}{ }^{a}(\%)$ \\
\hline dPS- $b$-P2VP/silica & $2.60 \pm 0.03$ & & $0.20 \pm 0.01$ & \\
UV/ozone treated & $0.178 \pm 0.005$ & $6.8 \pm 0.2$ & $0.012 \pm 0.005$ & $6.0 \pm 2.5$ \\
calcined, $400^{\circ} \mathrm{C}$ & $0.314 \pm 0.005$ & $12.1 \pm 0.2$ & $0.031 \pm 0.005$ & $15.3 \pm 2.5$
\end{tabular}

${ }^{a}$ Percentage of carbon and nitrogen remaining relative to the amounts in the dPS- $b$-P2VP/silica film.

are clearly evident in both the cross-linked template film and the silica/block copolymer hybrid film but have been eliminated to within the sensitivity limits of the measurement (ca. 5\%) following UV/ozone treatment or calcination. The peaks at 1430 and $1470 \mathrm{~cm}^{-1}$ arise from the P2VP component of the block copolymer as well and are also eliminated by both oxidative treatments. To determine quantitatively the degree of template removal, XPS measurements were performed on silica/ block copolymer films prior to and following oxidation, as summarized in Table 2. The films were etched to a depth of $\sim 20 \mathrm{~nm}$ below the free film surface prior to analysis to remove surface contamination and permit measurement of the composition of the film interior. Comparison of the ratios of carbon/silicon and nitrogen/ silicon before and after oxidation reveals that most of the polymer template species were removed, although measurable amounts of both carbon and nitrogen remained in the mesoporous silica films. For the UV/ ozone-treated samples, the ratios of C/Si and N/Si were $6-7 \%$ of their values in the initial block copolymer/silica film. Calcination to $400{ }^{\circ} \mathrm{C}$ was found to be less effective for removal of the template species, with the values of $\mathrm{C} / \mathrm{Si}$ and N/Si representing $12 \%$ and $15 \%$ of their initial values, respectively. These results indicate that the polymer template is not completely removed by UV/ ozone or thermal oxidation treatments. However, the levels of residual carbon are consistent with the amounts measured in previous XPS studies of the removal of lowmolecular-weight surfactant template species from mesoporous silica films via UV/ozone treatment and calcination. ${ }^{44}$ The remaining carbon and nitrogen may be present as species adsorbed to the interior mesopore surfaces or small fragments of the polymers occluded within the silica walls.

Variable-angle ellipsometry was used to determine the thicknesses and estimated porosities of the mesoporous silica films generated by UV/ozone treatment as functions of the degree of cross-linking of the initial polymer template (Table 1). As expected, the thicknesses of the films decreased with increases in the extent of cross-linking of the template film, since smaller volumes of silica were incorporated into the more highly crosslinked films. Even for the most lightly cross-linked template, the degree of contraction upon oxidation of the polymer template is larger than typically observed for mesoporous silica films formed via simultaneous assembly of silica and templating agent. ${ }^{45}$ Most likely this reflects a somewhat lower degree of silica condensation in the silica/dPS- $b$-P2VP hybrid films considered here. The refractive indices of the mesoporous silica films also increased with increased cross-linking of the template, from $n=1.361$ at $4.0 \%$ quaternization to $n=$ 1.427 at $19.5 \%$ quaternization. The porosities of the films can be estimated from these data using the Lorentz-Lorenz equation with an effective-medium approximation for the measured refractive indices $(n): 46$

$$
\frac{n^{2}-1}{n^{2}+2}=\epsilon \frac{n_{\mathrm{p}}{ }^{2}-1}{n_{\mathrm{p}}{ }^{2}+2}+(1-\epsilon) \frac{n_{\mathrm{w}}{ }^{2}-1}{n_{\mathrm{w}}{ }^{2}+2}
$$

The subscripts $\mathrm{p}$ and $\mathrm{w}$ denote the refractive indices of the mesopore channels and walls, respectively, and $\epsilon$ represents the volume fraction of the pores. Lowerbound values for the porosities of the films can be estimated by assuming a refractive indices of 1.456 for the silica matrix $\left(n_{\mathrm{w}}\right)$ and 1 for the pores $\left(n_{\mathrm{p}}\right) .{ }^{47}$ Values for the film porosities, estimated in this manner, are shown in Table 1; the porosities decrease with increased cross-linking of the dPS- $b$-P2VP template films. Mesoporous silica films prepared from block copolymer templates with degrees of cross-linking of $4.0 \%$ and $10.5 \%$ have estimated porosities of $18.6 \%$ and $13.4 \%$, respectively. Allowing for contraction of the silica framework during UV/ozone treatment, these values are reasonable, considering that the initial volume percent of the dPS cylinders responsible for the mesopores was $25 \%$. For the highest extent of cross-linking (19.5\%), an estimated porosity of only $5.5 \%$ was found, suggesting that the mesostructure of the film largely collapsed upon removal of the template polymer.

To establish the effects of template cross-linking on the morphologies of the mesoporous silica films, TEM was performed on UV/ozone-treated films that were prepared from films with different extents of P2VP quaternization. Cross-sectional TEM images are shown in Figure 7a,b,c of the mesoporous silica films that were prepared from the silica/polymer hybrid films whose TEM images appear in Figures 5f,h,j, respectively. Comparisons between the images in Figure 7 and those of the respective hybrid precursor films in Figure 5 show directly the contractions in thickness that occurred upon removal of the polymer templates. The mesoporous silica film prepared from a $4.5 \%$ quaternized template film, which showed a wrinkled texture in the hybrid silica/polymer precursor film (Figure 5f), is shown in Figure $7 \mathrm{a}$ to possess similarly undulating layers of cylinders after the polymer template species have been removed. Likewise, the mesoporous silica film prepared from a $9.5 \%$ quaternized template film (Figure 7b) shows an affinely distorted $2 \mathrm{D}$ hexagonal structure that is similar to that of the hybrid precursor (Figure 5h), though the structure has been compressed in the direction normal to the film plane upon removal of the block copolymer template. From the images in Figure $7 \mathrm{a}, \mathrm{b}$, the pore size is estimated to be about $10-12 \mathrm{~nm}$, which is near to the diameter of the dPS cylinders in the initial block copolymer template films $(12.6 \mathrm{~nm})$. In the case of the film prepared from the most heavily cross-linked template (25.7\% quaternization, Figure $7 \mathrm{c}$ ), a faint texturing on the length scale of the block copolymer remains, but it is not possible to resolve clearly defined mesopores. Apparently the amount of silica incorporated into this film was insufficient to form a robust mesoporous material, and hence removal of the template polymer species led to structural collapse. 

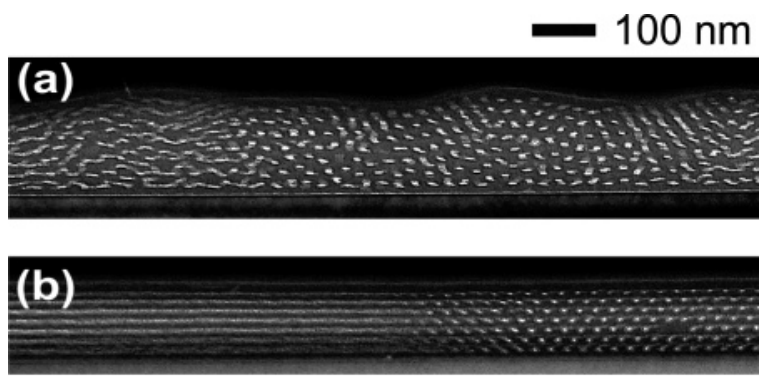

\section{(c)}

Figure 7. Cross-sectional TEM images of mesoporous silica films formed by UV/ozone treatment of the silica/dPS- $b$-P2VP films imaged in Figure 5f,h,j, with different extents of quaternization of the P2VP moieties: (a) $4.5 \%$, (b) 9.5\%, and (c) $25.7 \%$. The scale bar applies to all images.

Osmotic-Stress-Induced Wrinkling Instability. The wrinkling of the silica/block copolymer films formed from lightly cross-linked templates, as shown in Figure $5 \mathrm{~d}-\mathrm{g}$, has important implications for the approach presented here. It shows that under certain conditions osmotic stresses are sufficient to cause significant nonaffine distortions of the polymer mesostructure, even though the local organization of the structure is preserved by cross-linking. For many applications, such deformations may be detrimental to the material properties of the mesoporous inorganic films formed in this way. Conversely, if controllable, the instability could be a route to provide simultaneous patterning of substrates on two length scales: one dictated by the molecular weight and architecture of the block copolymer template species and the other by a balance between osmotic stresses and the mechanical properties of the polymer film. To better understand the nature of the instability, the influences of the anisotropic block copolymer mesostructure and film thickness on the length scale and morphology of the wrinkled structures are examined.

The wrinkling instability observed in the swelled silica/dPS- $b$-P2VP films appears to be closely related to the surface wrinkling instability that is known to occur upon swelling of confined isotropic hydrogels. ${ }^{15}$ Hybrid films of silica prepared under similar conditions but using cross-linked isotropic P2VP homopolymer template films also yielded wrinkled textures, indicating that the instability does not arise necessarily from mesostructural organization within the film. For the same thicknesses (100-200 nm) and extents of P2VP cross-linking $(\sim 2-4 \%)$ as block copolymer films that produced wrinkled textures, homopolymer films typically delaminated from the substrate during swelling due to insufficient adhesion. At slightly higher levels of cross-linking (5.9\%), the surfaces of the silica/block copolymer films remained flat (at least up to $170 \mathrm{~nm}$ initial film thickness), whereas the surfaces of silica/ homopolymer films had a wrinkled texture for films as thin as $130 \mathrm{~nm}$. SEM images of the silica/homopolymer film surfaces showed a series of creases spaced by a characteristic distance, as shown in Figure 8a,b. These features are similar in morphology to the creases seen during swelling of polyacrylamide gels first reported by Tanaka et al., ${ }^{15}$ though on a smaller length scale (as dictated by the smaller sample thickness). The wrinkled morphology varied from the cuneiform-like creases shown in Figure 8 to isolated linear creases, depending on the extent of cross-linking of the homopolymer P2VP
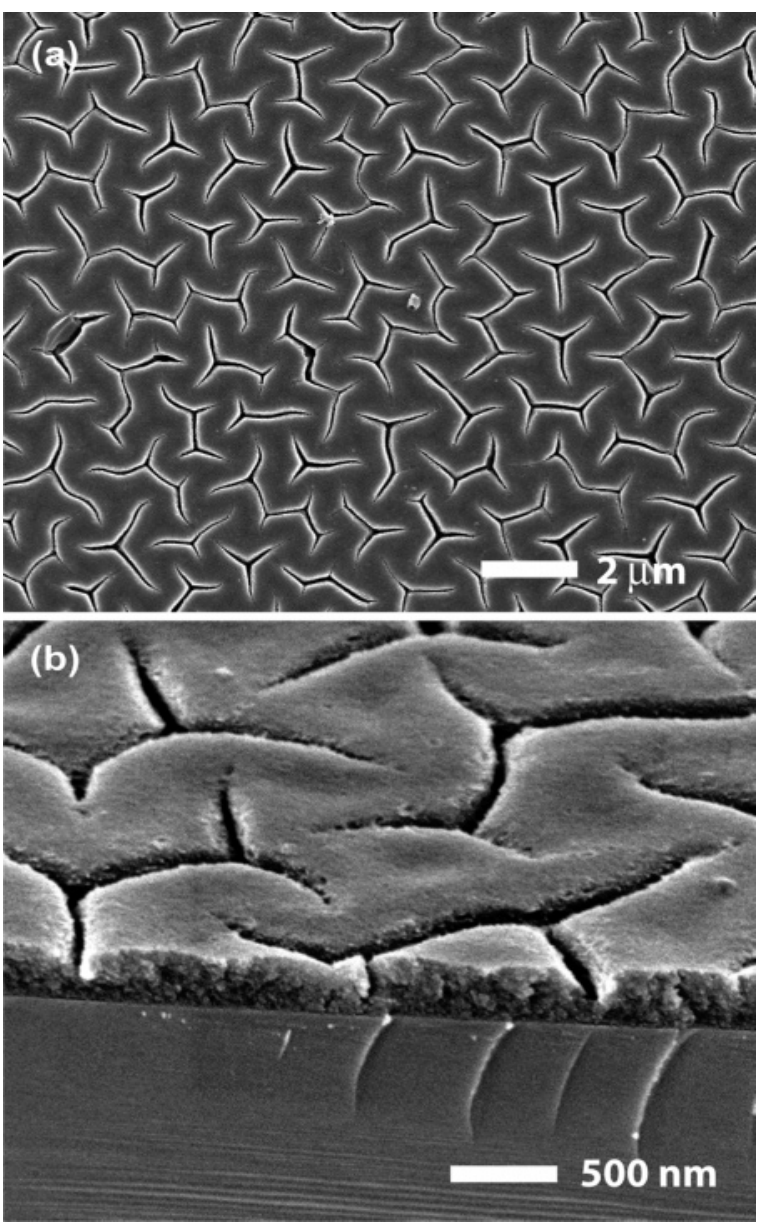

Figure 8. SEM images of wrinkled textures observed on the surface of a silica/P2VP homopolymer film prepared from a template initially $175 \mathrm{~nm}$ thick, with $5.9 \%$ P2VP quaternization. (a) Plan-view image showing the in-plane morphology of the wrinkles and (b) cross-sectional image of the same film obtained by tilting the sample to $60^{\circ}$ and imaging an edge cleaved through the middle of the sample.

template film and the amount of silica that was incorporated. The characteristic length scale $(\lambda)$ of the wrinkling instability of the silica/homopolymer films was determined by taking the azimuthally averaged magnitude of the 2D Fourier transform of a series of SEM images for films with different initial thicknesses (l). As shown in Figure 9, the scaling of the characteristic crease spacing as a function of film thickness could be fit by a power law $\lambda=l^{a}$ with an exponent of $a=$ $1.08 \pm 0.06$. This is consistent with the findings of Tanaka et al., ${ }^{15}$ who reported a linear relationship between the characteristic length scale of wrinkling and the thickness of the swelled gel layers.

For the mesostructured hybrid silica/block copolymer films, the wrinkling instability and spacing also depend on the initial film thickness. Figure 10 shows SEM images of a series of wrinkled silica/block copolymer films with different initial thicknesses, all with the same extent of cross-linking (3.5\%). Alongside each image is shown the azimuthally averaged magnitude of its $2 \mathrm{D}$ Fourier spectrum. The top image (Figure 10a) corresponds to an initial film thickness of $110 \mathrm{~nm}$ (5 layers of dPS cylinders); below this thickness, otherwise identical films showed no evidence of wrinkling. Apparently, for a given degree of cross-linking, there is a minimum thickness below which the osmotic stresses developed in the film are insufficient to give rise to the wrinkling 


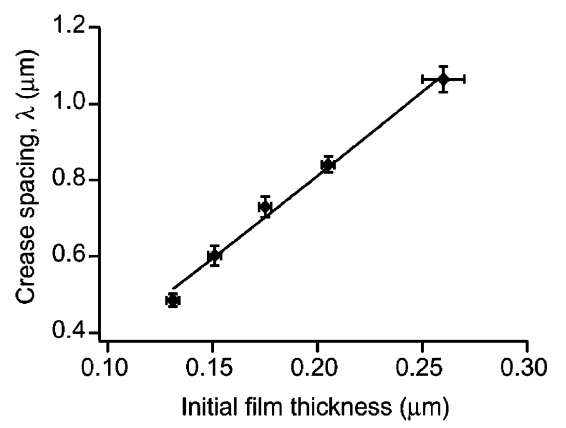

Figure 9. Dependence of the characteristic length scale of surface wrinkling in silica/P2VP films for samples prepared from templates with 9.1\% P2VP quaternization and a range of initial P2VP film thicknesses. The solid line corresponds to a power-law fit to the data with an exponent of $1.08 \pm 0.06$. The data point at a thickness of $0.26 \mu \mathrm{m}$ corresponds to an image taken at the edge of the next thinnest sample, where an excess of material was deposited during spin-coating. The initial film thickness in this region was inferred from the ratio of the composite silica/polymer film thicknesses measured by cross-sectional SEM.
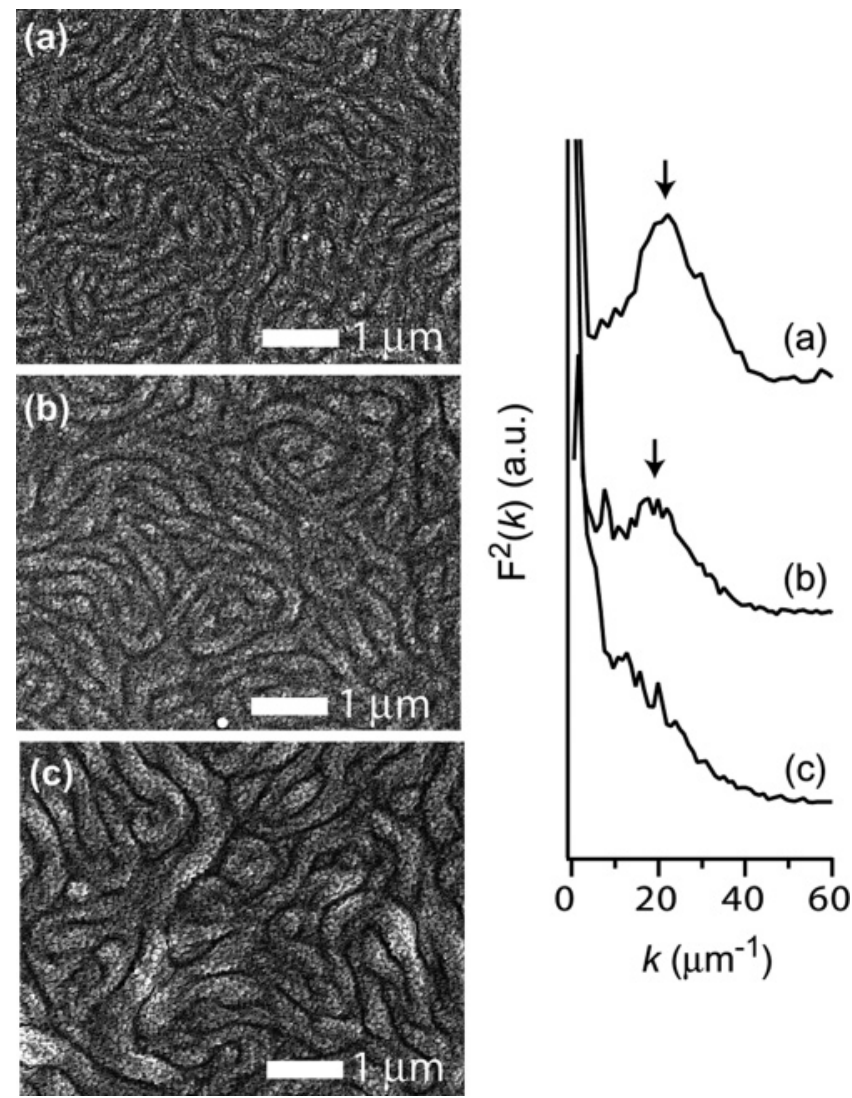

Figure 10. (left) SEM images of surface wrinkling in silica/ dPS- $b$-P2VP films formed from block copolymer template films with 3.5\% quaternization and different initial thicknesses: (a) $110 \mathrm{~nm}$ thick (5 layers of dPS cylinders), (b) $130 \mathrm{~nm}$ (6 layers of cylinders), and (c) $150 \mathrm{~nm}$ (7 layers of cylinders). (right) Azimuthal averages of the magnitudes of the 2D Fourier transforms of the images at left, where $k=2 \pi / \lambda$, with $\lambda$ as the real-space length. Peaks in the Fourier spectra (denoted by arrows) can be seen at (a) $21.7 \pm 0.3 \mu \mathrm{m}^{-1}$ (corresponding to $290 \pm 3 \mathrm{~nm}$ ) for the 5-layer sample and (b) $18.2 \pm 0.3 \mu \mathrm{m}^{-1}$ $(346 \pm 6 \mathrm{~nm})$ for the 6-layer sample. (c) For the 7-layer sample, there is no resolvable peak in the Fourier spectrum. For 4 dPS cylinder layers, no wrinkling was observed, whereas for 8 layers extensive film delamination and buckling occurred.

instability. The Fourier spectrum for the 5-layer-thick film in Figure 10a shows a clear maximum value

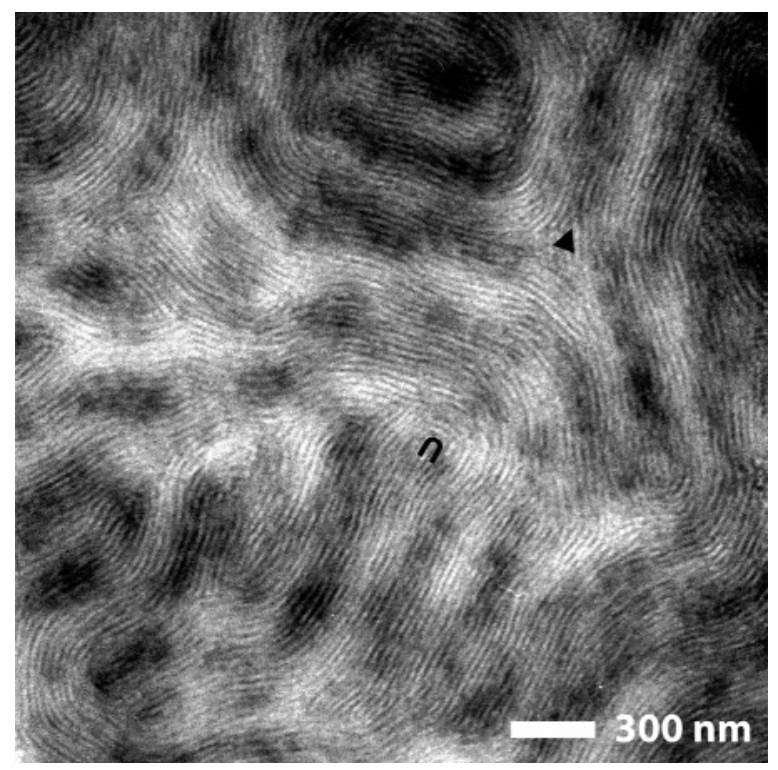

Figure 11. Plan-view TEM image of the same surfacewrinkled film as in Figure 10a, with an initial thickness of $110 \mathrm{~nm}$ (5 layers of dPS cylinders) and 3.5\% quaternization of the template film. The approximate locations of a $+1 / 2$ disclination $(\cup)$ and $a-1 / 2$ disclination $(\boldsymbol{\Delta})$ are marked.

associated with a characteristic length scale for the wrinkles centered at $290 \pm 3 \mathrm{~nm}$. For the 6-layer film $(130 \mathrm{~nm})$, the wrinkles were more easily visible due to their increased amplitude. There is still a maximum value in the Fourier spectrum, corresponding to a longer length scale $(346 \pm 6 \mathrm{~nm})$, though the peak is more difficult to resolve, presumably due to a broader distribution of wrinkle length scales in the image. For a film of 7 layers $(150 \mathrm{~nm})$, the amplitude and characteristic spacing of the features had both clearly increased, but there is no longer a resolvable peak in the Fourier spectrum. Correspondingly, it can be seen by eye that the wrinkled morphology consists of a broader distribution of feature sizes. Above 7 layers in thickness, osmotic stresses were sufficient to produce localized delamination and buckling of the film (see below), which changed the character of the surface texture. While the results presented here are consistent with a linear dependence of wrinkle spacing on film thickness, the data are insufficient to establish this conclusively. To examine quantitatively the scaling of this instability with film thickness, large monodomains of the $2 \mathrm{D}$ hexagonal structure are required in order to eliminate the influence of the block copolymer defect structure on the morphology and wavelength of the wrinkling. In addition, it will be important to improve adhesion of the polymer film to the substrate to allow characterization of wrinkling in thicker films at low degrees of crosslinking without delamination.

While the wrinkling instabilities observed in dPS- $b$ P2VP/silica and P2VP homopolymer/silica films appear to be related, the morphologies of wrinkling in the two cases are quite different. In contrast to the sharp creases formed on the surfaces of wrinkled silica/homopolymer films (Figure 8), the patterns observed on the surfaces of wrinkled silica/block copolymer films (Figures 5 and 10) resemble a coarse-grained version of the fingerprint defect pattern of the block copolymer itself. This suggests that, in the case of the dPS- $b$-P2VP/silica films, the texture of the wrinkling is directed by the cylindrical mesostructure of the block copolymer. Figure 11 shows 


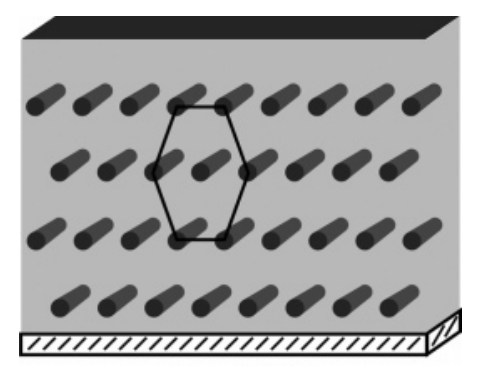

$\uparrow$ Affine swelling
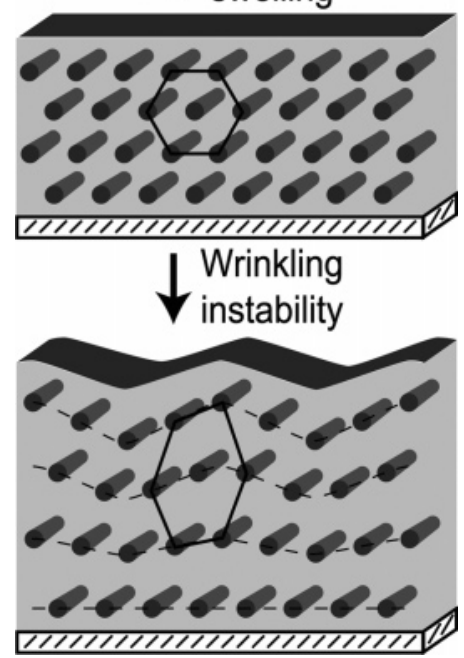

Figure 12. Schematic diagram depicting the proposed deformation of the block copolymer mesostructure for the cases of affine swelling and the osmotic-stress-induced wrinkling instability.

a TEM micrograph taken through a section of the film from Figure 10a (initially $110 \mathrm{~nm}$ thick with an extent of quaternization of $3.5 \%$ ). In this case, the thickness was chosen to be just above that for the onset of wrinkling since larger amplitude distortions obscured the relationship between the mesostructure and the wrinkled morphology, and thicker films did not easily permit TEM imaging. The wrinkling pattern is evident as a series of large lighter and darker stripes of periodicity $\sim 300 \mathrm{~nm}$. From this image, it can clearly be seen that the long axes of the dPS cylinders (the smaller stripes with periodicity $\sim 20 \mathrm{~nm}$ ) run parallel to the ridges and valleys of the wrinkled texture. Correspondingly, the direction of wrinkling changes direction in the vicinity of defects in the block copolymer structure, such as the $+1 / 2$ and $-1 / 2$ disclinations marked in Figure 11. Thus, it is clear that the texture of the surface wrinkling is derived from the anisotropic mesostructure of the block copolymer.

A schematic illustration of the proposed deformation of the block copolymer film is shown in Figure 12 for the cases of affine swelling and the wrinkling instability. The mechanical properties of the swelled cross-linked block copolymer film are anisotropic on a length scale that is smaller than the average distance between defects in the block copolymer mesostructure (ca. 500 $\mathrm{nm}$ ), with the modulus along the direction parallel to the axes of the glassy dPS cylinders being as much as 4 orders of magnitude larger than that of the P2VP hydrogel in the perpendicular directions. ${ }^{48} \mathrm{~A}$ relative translation of the cylinders transverse to their long axes, as diagrammed in Figure 12, involves only changes in the elastic energy of the P2VP hydrogel surrounding the
dPS cylinders, without any elastic deformation of the cylinders themselves. Such a deformation allows regions of the film to expand "laterally", thus helping to alleviate the in-plane osmotic stresses. Consider the deformation of a unit cell of the initial block copolymer mesostructure, as indicated by the hexagons drawn in Figure 12. Affine swelling (Figure 12, top) leads to an expansion of the structure normal to the film plane, while the inplane spacing between neighboring cylinders is maintained at the melt value. By wrinkling in the manner diagrammed in Figure 12, bottom, the spacing between a cylinder and each of its neighbors is expanded more uniformly. In this respect, the instability is quite different than the undulation of cylindrical microdomain block copolymers that is observed under uniaxial strain; ${ }^{37}$ in that case, the structure deforms along the cylinder axes in order to prevent the spacing between neighboring cylindrical microdomains from having to change extensively. Here, the structure wrinkles with little deformation along the cylinder axes to allow the spacings between neighboring microdomains to increase more uniformly than in the case of uniaxial swelling.

Macroscopic Film Buckling. Formation of the wrinkled surface texture discussed above depends on the film remaining anchored to the substrate during swelling. If the film is able to detach from the substrate, it can form macroscopic buckles that allow lateral swelling to relieve the in-plane osmotic stresses. It is well-known that in thin films under biaxial compression film delamination giving rise to macroscopic buckling can be observed once $G$, the strain energy released per unit area crack advance at the interface, exceeds the adhesive fracture energy of the interface $G_{c},{ }^{39}$ including the case of swelled polymer films. ${ }^{17,18}$ If the biaxial compressive stress $\sigma$ is uniform through the thickness, $G$ is proportional to $\sigma^{2} l$, and buckling via interface debonding will occur for films above the thickness where $G=G_{\mathrm{c}}{ }^{49}$ The SEM micrograph in Figure 13a shows the surface of a silica/dPS- $b$-P2VP film prepared from a template with $3.5 \% \mathrm{P} 2 \mathrm{VP}$ quaternization. The initial thickness of this film was between that required to form 6 and 7 layers of cylinders, so islands with a thickness corresponding to 7 layers $(150 \mathrm{~nm})$ are seen on a background of film with a thickness corresponding to 6 layers $(130 \mathrm{~nm})$. In Figure $13 \mathrm{a}$, the region to the left side of the image is 6 layers of dPS cylinders thick, whereas the remainder is 7 layers. The approximate location of the interface between the regions is indicated by a dotted line. At 6 layers, the film showed a uniform wrinkled texture across the entire sample surface. At 7 layers, however, isolated buckles were observed, such as the one denoted with an arrow. Tilting the sample indicated that these buckles protruded to a height several times the thickness of the surrounding swelled film. Thus, it can be concluded that these buckles correspond to localized delamination of the film from the substrate, presumably due to weaker adhesion at the locus of buckling, compared to the surrounding regions. An increase of the film thickness to 9 layers of cylinders (190 nm) led to much more extensive buckling, as shown in the SEM micrograph in Figure 13b. Further increases in film thickness lead to a progressive coarsening of the buckling texture, eventually leading to complete delamination of the film from the substrate.

Figure 13c shows a TEM image of a cross section of a buckled region of a silica/polymer film that was prepared so that the electron beam was incident nearly 

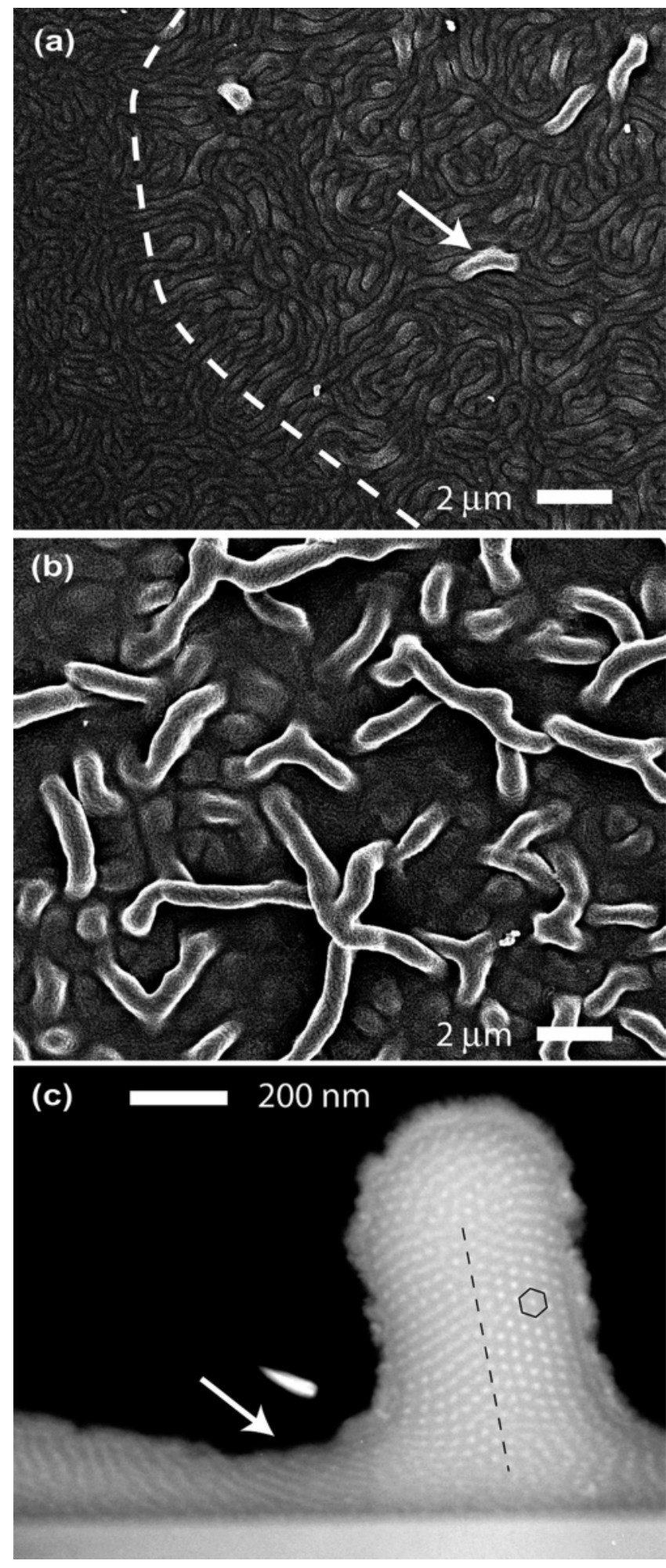

Figure 13. SEM images of buckles in silica/dPS- $b$-P2VP films prepared from template films with 3.5\% P2VP quaternization. (a) The region to the left of the dotted line corresponds to 6 layers of dPS cylinders and shows no buckling, whereas the region to the right corresponds to 7 layers and shows isolated buckles, as indicated by the arrow. (b) At 9 layers of dPS cylinders, the buckling is much more pronounced. (c) Crosssectional TEM image of an isolated buckle similar to that observed in (a), in a film formed from a template with $1.6 \%$ P2VP quaternization and a thickness of $7 \mathrm{dPS}$ cylinder layers. The approximate location of the original film/substrate interface for the buckled material is shown as a dotted line.

parallel to the long folding direction of the buckle. The template film was $150 \mathrm{~nm}$ thick (7 layers of dPS cylinders), with a degree of P2VP quaternization of $1.6 \%$. The resulting silica/polymer film showed a wrinkled texture with isolated buckles similar to those in Figure 13a. The approximate location of the original film/ substrate interface is shown by a dotted line in Figure 13c. Upon buckling, the interface appears to have folded upon itself, as apparently the section of film became detached from the interface and was compressed from the sides, due to the osmotic stresses in the surrounding regions of the film. The location of the original interface can be determined by identifying 7 layers of cylinders on either side of it. In addition, tilting experiments reveal that the cylinders on the left side of the dotted line are oriented collectively in a slightly different direction than those on the right, confirming that these areas correspond to initially disparate areas of the film. In both cases, however, the cylinder axes are running very nearly parallel to the long folding direction of the buckle. As in the case of the wrinkling instability, this allows the cylinders to space themselves apart more uniformly and requires little elastic deformation of the cylinders. Examination of the intercylinder spacing within the buckle shows that the cylinders are very nearly uniformly spaced; for example, a regular hexagon has been drawn around one dPS cylinder in Figure 13c to show that buckling has allowed the mesostructure to regain nearly 6-fold symmetry on a local scale. Interestingly, the horizontal regions of the film just to the sides of the buckle (as indicated by an arrow) are considerably thinner than the surrounding areas, suggesting that plastic deformation of these regions has occurred during buckle formation. Presumably, upon initiation of a crack at the interface between the film and the substrate, the buckle is forced away from the substrate, as material from the surrounding region expands laterally to relieve osmotic stresses. Meanwhile, the film/substrate crack continues to propagate laterally, freeing more of the film to expand into the buckled region. However, at some point the crack encounters a disclination in the block copolymer structure that then leads to a change in the direction of the cylindrical microdomains. At this point, additional growth of the buckle becomes less favorable, since deformation of the glassy dPS cylinders would be required. Apparently, the energy released during formation of this crack is enough to plastically deform the cylinders over a short distance, leading to the thinned region surrounding the buckle; however, buckle formation is ultimately halted.

Implications of Swelling-Induced Instabilities. Under the proper conditions, it is possible to use crosslinked dPS- $b$-P2VP block copolymer films to produce mesoporous silica replicas that closely mimic the mesostructures of the initial template films. Figure 14a shows a TEM micrograph of such a mesoporous silica film and the location of the template film in the parameter space defined by thickness and extent of cross-linking of the template (Figure 14b). However, the wrinkling and buckling instabilities discussed above present important limitations to the approach presented here. On the parameter-space diagram in Figure 14b, approximate boundaries are drawn for the regions that correspond to these two types of instabilities.

To suppress film delamination, the samples considered here were all prepared on amine-functionalized silicon substrates. The choice of amine functionality was made to allow covalent grafting of the polymer layer to 
(a)

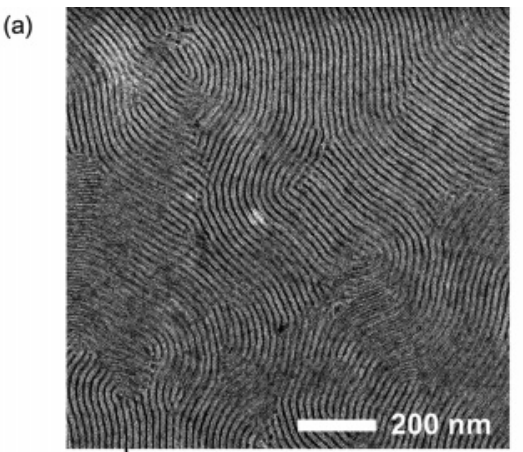

(b)

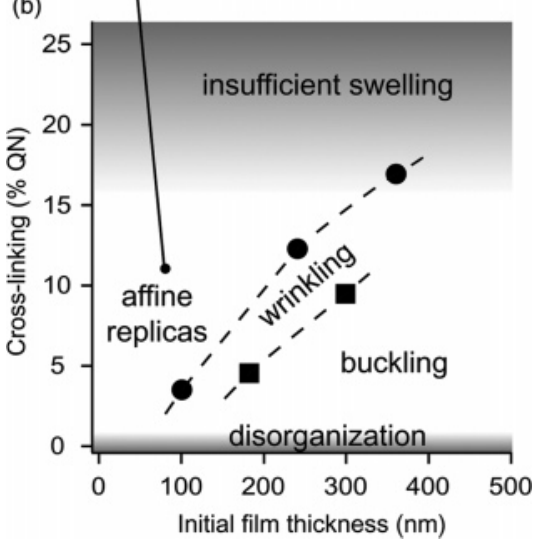

Figure 14. (a) Plan-view TEM image of a mesoporous silica film that is essentially an affinely distorted replica of the initial dPS- $b$-P2VP template film (4 layers of dPS cylinders thick with $11.1 \%$ P2VP quaternization). (b) The investigated region of parameter space defined by the thicknesses and extents of P2VP cross-linking of dPS- $b$-P2VP template films. Approximate locations of the boundaries for the two instabilities observed here are indicated by the dashed lines.

the substrate via the same DIB vapor treatment used to cross-link the polymer. While we have not established the degree to which covalent bonds are formed between the film and the substrate, films that were prepared on amine-functionalized silicon wafers generally did not delaminate until greater thicknesses than equivalent samples on nonfunctionalized silicon. In the best case, the presence of the aminosilane layer at the surface increased the thickness for the onset of delamination by approximately a factor of 2 . However, the level of substrate adhesion varied considerably between aminefunctionalized wafers prepared at different times, presumably due to the sensitivity of the structure of the silane layer to the deposition conditions, especially humidity. The line drawn in Figure 14b to indicate the onset of buckling shows the worst-case scenario for the amine-functionalized surfaces used here, i.e., the minimum film thickness at which extensive delamination was observed for a given degree of cross-linking. While we did not conduct a detailed study of the impact of the silane layer on delamination, it is clear that improvements in adhesion between the block copolymer film and the substrate can effectively be used to suppress delamination and thus buckling. The ideal approach would allow independent control over grafting of the polymer layer to the substrate and the degree of cross-linking of the polymer network.

Suppression of the wrinkling instability is less straightforward, and in practice this instability places an upper limit on the thickness of template films to which the technique presented here can be applied. In the present work, cross-linking of $\sim 10 \%$ has been shown to yield films that remain planar for initial thicknesses of up to $\sim 200 \mathrm{~nm}$. The onset of wrinkling can be extended to greater thickness by increasing the degree of crosslinking, as shown by the circles in Figure 14b, which represent the minimum thicknesses at which clear undulation of the cylinder layers were observed. Increasing the extent of cross-linking simultaneously increases the elastic moduli of the film and decreases the magnitude of the in-plane swelling stresses. Apparently, beyond a certain extent of cross-linking, the elastic energy penalty associated with wrinkling exceeds the favorable change in energy due to alleviation of lateral swelling stresses, and the film deforms affinely. It is important to note that for the more extensively cross-linked dPS- $b$-P2VP template films investigated here the characteristic length scale of the observed wrinkling instability was larger than the average distance between defects in the block copolymer mesostructure. In this case, the film is expected to behave more similarly to an isotropic material, meaning that the deformation shown schematically in Figure 12 is no longer expected to be valid, and significant plastic deformation of the dPS cylinders may take place. An interesting avenue for further investigation is the dependence of the texture of this wrinkling instability on the domain size of the block copolymer.

The approach of cross-linking the polymer template species more extensively to suppress wrinkling can only be taken so far before the film no longer swells sufficiently to incorporate sufficient inorganic material to form a robust network. Here, cross-linking of the initial template to an extent of 20-26\% P2VP quaternization was too large to form stable mesoporous films. This regime is indicated by the gray area at the top of the parameter-space diagram in Figure 14b. The point at which cross-linking of the template becomes too extensive will depend on the processing conditions used and how effectively and homogeneously the swelled hydrophilic regions of the polymer template film can be filled with inorganic material. In any case, the production of planar films thicker than a few hundred nanometers will most likely require modifications to the current technique. For example, one can envision the formation of cross-linked template films that have removable organic species ${ }^{50-52}$ mixed into the majority component of the block copolymer. It may then be possible to replace the removable component with a sufficient volume of inorganic precursor species without having to swell the film extensively beyond the thickness at the point of cross-linking.

\section{Conclusions}

We have investigated the role of P2VP cross-linking on the morphologies of silica/polymer hybrid films and mesoporous silica films formed by swelling of preformed poly $\left(d_{8}\right.$-styrene-block-2-vinylpyridine) template thin films. Without cross-linking, extensive reconstruction of the copolymer mesostructure took place during swelling, effectively nullifying the inherent advantage of this approach, namely being able to preorganize the template. At low to moderate degrees of cross-linking $(\leq 10 \%$ of the P2VP units quaternized by 1,4-diiodobutane), the films swelled extensively in acidic aqueous environments, allowing incorporation of sufficient amounts of silica into the hydrophilic P2VP region to ultimately form stable mesoporous silica films. For sufficiently thin films, this swelling occurred in an essentially affine 
manner, leading to hybrid silica/polymer replicas of the initial block copolymer templates. However, for films that were too thick, osmotic stresses were sufficient to produce a wrinkling instability that is closely related to the instability observed during the swelling of confined isotropic hydrogels. ${ }^{15}$ The minimum thickness at which surface wrinkling occurred increased as the extent of P2VP cross-linking of the template films was increased. For the films considered here, which were only slightly above the minimum thickness required for the wrinkling instability to occur, the cylindrical microdomain structure of the block copolymer served to direct the morphology of the wrinkles. In addition, the onset of the wrinkling instability was suppressed relative to homopolymer films of the same degree of crosslinking and equivalent thickness of P2VP.

For a given dPS- $b$-P2VP film thickness, the wrinkling instability observed here can be prevented by sufficiently cross-linking the P2VP component of the initial template. However, the amount of silica incorporated into the swelled template film decreases with increased P2VP cross-linking and eventually becomes insufficient to form a robust silica network or a stable mesoporous material. In such cases, this leads to a collapse of mesostructural order upon removal of the polymer template species. Thus, the formation of inorganic materials that accurately replicate the mesostructure of the block copolymer template film depends on the ability to strike a balance between osmotic-stressinduced deformations and the need for sufficient swelling to incorporate adequate amounts of the networkforming inorganic material. An extent of P2VP crosslinking of $\sim 10 \%$ was found to effectively balance these considerations for the film thicknesses considered here (100-200 nm), leading to mesostructured and mesoporous silica films with morphologies that were closely related to those of the initial block copolymer template. To extend the current approach to significantly thicker template films, it will be necessary to either reduce the magnitude of the osmotic swelling stresses generated or modify the mechanical properties of the polymer film to reduce its tendency to form surface wrinkles. Developing a more complete understanding of the instabilities that occur upon swelling of cross-linked block copolymer films will be important for the effective use of these materials as templates for mesostructured inorganic films.

Acknowledgment. We thank Dr. Ryan Toomey and Dr. Alejandro Parra for helpful discussions and assistance with ellipsometry, Jayna Jones and Dr. Uri Raviv for performing the SAXS measurement, Dr. Tom Mates for help with SIMS and XPS, and Dr. Tim Alig for assistance with SFM. We also gratefully acknowledge Professors Glenn Fredrickson, Tony Evans, Leo Radzihovsky, Phil Pincus, and Courtney Lannert and Dr. Christian Santangelo for their helpful comments regarding mechanical instabilities during swelling of confined hydrogels. Portions of this research were carried out at the Stanford Synchrotron Radiation Laboratory, a national user facility operated by Stanford University on behalf of the U.S. Department of Energy, Office of Basic Energy Sciences. R.H. acknowledges support from the U.S. National Science Foundation for a Graduate Research Fellowship and the Corning Foundation for a Fellowship in Materials Research. This work was supported by the MRSEC Program of the National Science Foundation under Award DMR-00-
80034. Additional support was provided through the National Science Foundation Division of Materials Research under Award DMR-02-33728.

\section{References and Notes}

(1) Pai, R. A.; Humayun, R.; Schulberg, M. T.; Sengupta, A.; Sun, J. N.; Watkins, J. J. Science 2004, 303, 507-510.

(2) Tanaka, S.; Nishiyama, N.; Oku, Y.; Egashira, Y.; Ueyama, K. J. Am. Chem. Soc. 2004, 126, 4854-4858.

(3) Hayward, R. C.; Chmelka, B. F.; Kramer, E. J. Adv. Mater., in press.

(4) Lu, Y. F.; Ganguli, R.; Drewien, C. A.; Anderson, M. T.; Brinker, C. J.; Gong, W. L.; Guo, Y. X.; Soyez, H.; Dunn, B.; Huang, M. H.; Zink, J. I. Nature (London) 1997, 389, 364368.

(5) Zhao, D.; Yang, P.; Melosh, N.; Feng, J.; Chmelka, B. F.; Stucky, G. D. Adv. Mater. 1998, 10, 1380-1385.

(6) Soler-Illia, G. J. de A. A.; Crepaldi, E. L.; Grosso, D.; Sanchez, C. Curr. Opin. Colloid Interface Sci. 2003, 8, 109-126.

(7) Morkved, T. L.; Lu, M.; Urbas, A. M.; Ehrichs, E. E.; Jaeger, H. M.; Mansky, P.; Russell, T. P. Science 1996, 273, 931933.

(8) Thurn-Albrecht, T.; Schotter, J.; Kastle, C. A.; Emley, N.; Shibauchi, T.; Krusin-Elbaum, L.; Guarini, K.; Black, C. T.; Tuominen, M. T.; Russell, T. P. Science 2000, 290, 21262129.

(9) Segalman, R. A.; Yokoyama, H.; Kramer, E. J. Adv. Mater. 2001, 13, 1152-1155.

(10) Kim, S. H.; Misner, M. J.; Xu, T.; Kimura, M.; Russell, T. P. Adv. Mater. 2004, 16, 226-231.

(11) Flory, P. J.; Rehner, J. J. Chem. Phys. 1943, 11, 521-526.

(12) Toomey, R.; Freidank, D.; Rühe, J. Macromolecules 2004, 37, $882-887$.

(13) Seelenmeyer, S.; Deike, I.; Rosenfeldt, S.; Norhausen, C.; Dingenouts, N.; Ballauff, M.; Narayanan, T.; Lindner, P. J. Chem. Phys. 2001, 114, 10471-10478.

(14) Harmon, M. E.; Jakob, T. A. M.; Knoll, W.; Frank, C. W. Macromolecules 2002, 35, 5999-6004.

(15) Tanaka, T.; Sun, S. T.; Hirokawa, Y.; Katayama, S.; Kucera, J.; Hirose, Y.; Amiya, T. Nature (London) 1987, 325, 796798.

(16) Teschke, O.; Kleinke, M. U.; Galembeck, F. J. Appl. Polym. Sci. 1995, 57, 1567-1573.

(17) Sharp, J. S.; Jones, R. A. L. Phys. Rev. E 2002, 66, 011801.

(18) Sharp, J. S.; Jones, R. A. L. Adv. Mater. 2002, 14, 799-802.

(19) Yokoyama, H.; Mates, T. E.; Kramer, E. J. Macromolecules 2000, 33, 1888-1898

(20) Lim, H.; Lee, Y.; Han, S.; Yoo, Y.; Kim, K.-J. Bull. Korean Chem. Soc. 1999, 20, 853-856.

(21) Fasolka, M. J.; Harris, D. J.; Mayes, A. M.; Yoon, M.; Mochrie, S. G. J. Phys. Rev. Lett. 1997, 79, 3018-3021.

(22) Jasperson, S. N.; Schnatterly, S. E. Rev. Sci. Instrum. 1969, 40, 761-767.

(23) The refractive index varies slightly at high concentrations of $\mathrm{HCl}$, but correcting for this effect led to only small changes in the fitted film thicknesses $(\sim 1-2 \mathrm{~nm})$.

(24) Azzam, R. M. A.; Bashara, N. M. Ellipsometry and Polarized Light; North-Holland Pub. Co.: Amsterdam, 1977.

(25) Beaglehole, D. Physica B \& C 1980, 100, 163-174.

(26) Guinier, A.; Fournet, G. Small-Angle Scattering of X-rays; Wiley: New York, 1955.

(27) Saito, R.; Okamura, S.; Ishizu, K. Polymer 1992, 33, 10991101.

(28) Barber, M.; Connor, J. A.; Guest, M. F.; Hillier, I. H.; Schwarz, M. J. Chem. Soc., Faraday Trans. 2 1973, 551-558.

(29) It is important to note that the degree of cross-linking reported in terms of percentage of quaternary nitrogen atoms is not equivalent to the fraction of monomers participating in cross-links. It is expected that the majority of DIB molecules quaternize only one pyridine unit and hence do not form cross-links. However, it is difficult to obtain a reliable estimate of how large this fraction is. Thus, the degree of quaternization represents a relative indicator of the extent of cross-linking and not an absolute measure.

(30) FTIR measurements on cross-linked block copolymer films soaked in acidic solutions indicated that the effective $\mathrm{p} K$ for the pyridine groups in the films was approximately 2.5 .

(31) Krämer, E.; Förster, S.; Göltner, C.; Antonietti, M. Langmuir 1998, 14, 2027-2031. 
(32) Cho, G.; Jang, J.; Jung, S.; Moon, I. S.; Lee, J. S.; Cho, Y. S.; Fung, B. M.; Yuan, W. L.; O’Rear, E. A. Langmuir 2002, 18, $3430-3433$.

(33) Fujita, N.; Asai, M.; Yamashita, T.; Shinkai, S. J. Mater. Chem. 2004, 14, 2106-2114.

(34) Hahn, J.; Webber, S. E. Langmuir 2004, 20, 4211-4219.

(35) The amount of silica incorporated into the film depends on the length of exposure to the precursor solution as well as the $\mathrm{pH}$ and concentration of the solution. The conditions employed here have not been extensively optimized but were sufficient to yield stable mesoporous films at low to moderate extents of cross-linking.

(36) de Gennes, P. G.; Prost, J. The Physics of Liquid Crystals; Oxford University Press: New York, 1993.

(37) Honeker, C. C.; Thomas, E. L. Chem. Mater. 1996, 8, 17021714.

(38) A similar wrinkling instability was observed from SFM images of the surface of a cross-linked block copolymer film swelled under acidic water in the absence of silica sol-gel precursors (data not shown). Thus, the observed rippling seems to be induced primarily by the osmotic swelling stresses developed in the film rather than by incorporation of silica precursor species. The manner in which incorporation of silica into the block copolymer structure may impact the morphology of the wrinkling by altering the balance between elastic energy and osmotic stresses is an area for future investigation.

(39) Freund, L. B.; Suresh, S. Thin Film Materials; Cambridge University Press: Cambridge, 2003.

(40) Strictly speaking, the deformation is not truly affine since the glassy dPS cylinders do not swell normal to the substrate, implying a degree of nonaffine deformation in their vicinity. Considering the relative positions of the central axes of the cylinders, however, the structures of the composite silica/ polymer films in Figure $5 \mathrm{~h}, \mathrm{j}$ appear to be related to the initial structure of the block copolymer template via a uniform expansion normal to the film plane.

(41) The spacing between layers of cylinders in the more highly cross-linked sample is less extensively dilated by silica, as seen also from ellipsometric measurements of total film thickness (Table 1). No attempt has been made to quantify the extent of swelling from the cross-sectional TEM images, however, since some contraction of the structure normal to the film plane typically occurred during FIB sample preparation, presumably due to ion beam or electron beam damage.

(42) Clark, T.; Ruiz, J. D.; Fan, H.; Brinker, C. J.; Swanson, B. I.; Parikh, A. N. Chem. Mater. 2000, 12, 3879-3884.

(43) Hozumi, A.; Yokogawa, Y.; Kameyama, T.; Hiraku, K. Sugimura, H.; Takai, O.; Okido, M. Adv. Mater. 2000, 12, 985-987.

(44) Hozumi, A.; Sugimura, H.; Hiraku, K.; Kameyama, T.; Takai, O. Chem. Mater. 2000, 12, 3842-3847.

(45) Alberius, P. C. A.; Frindell, K. L.; Hayward, R. C.; Kramer, E. J.; Stucky, G. D.; Chmelka, B. F. Chem. Mater. 2002, 14 $3284-3294$

(46) Born, M.; Wolf, E. Principles of Optics; Cambridge University Press: Cambridge, 1999.

(47) This approach leads to a systematic underestimation of the pore volume since the adsorption of atmospheric water by the mesopores increases the effective refractive index of the pore channels. Upon removal from the UV/ozone chamber, adsorption of significant amounts of water was clearly observed to occur from a change in the interference colors of the porous films

(48) The Young's modulus of glassy bulk polystyrene is ca. $3 \mathrm{GPa}$, while the modulus of the P2VP hydrogel is estimated to be $\sim 300 \mathrm{kPa}$, based on a simple Flory-Rehner treatment, assuming that the density of elastically active cross-links is $\sim 1 \%$ of the density of P2VP monomers. It is important to note that in the regime investigated here the osmotic stresses are apparently insufficient to produce breakup of the cylinders via plastic deformation of the dPS component.

(49) In the case of the swelled cross-linked block copolymer films investigated here, the wrinkling instability relieves some of the compressive stress near the free interface, meaning that the stress is not uniform throughout the film thickness. Nonetheless, the strain energy release rate increases with thickness, leading to debonding of films above a certain thickness.

(50) Liu, G.; Ding, J.; Guo, A.; Herfort, M.; Bazett-Jones, D. Macromolecules 1997, 30, 1851-1853.

(51) Mäki-Ontto, R.; de Moel, K.; de Odorico, W.; Ruokolainen, J.; Stamm, M.; ten Brinke, G.; Ikkala, O. Adv. Mater. 2001 $13,117-121$.

(52) Sidorenko, A.; Tokarev, I.; Minko, S.; Stamm, M. J. Am. Chem. Soc. 2003, 125, 12211-12216.

MA0477854 